Draft VERSiOn June 18, 2020

Typeset using $\mathrm{LAT}_{\mathrm{E}} \mathrm{X}$ twocolumn style in AASTeX63

\title{
Populating the Black Hole Mass Gaps In Stellar Clusters: General Relations and Upper Limits
}

\author{
Johan SAmsing (D) ${ }^{1}$ And Kenta Hotokezaka ${ }^{2,3}$ \\ ${ }^{1}$ Niels Bohr International Academy, The Niels Bohr Institute, \\ Blegdamsvej 17, 2100 Copenhagen, Denmark. \\ ${ }^{2}$ Department of Astrophysical Sciences, Princeton University, \\ Peyton Hall, 4 Ivy Lane, Princeton, NJ 08544, USA. \\ ${ }^{3}$ Research Center for the Early Universe, Graduate School of Science, \\ University of Tokyo, Bunkyo-ku, Tokyo 113-0033, Japan.
}

Submitted to ApJ

\begin{abstract}
Theory and observations suggest that single-star evolution is not able to produce black holes (BHs) with masses in the range $3-5 M_{\odot}$ and above $\sim 45 M_{\odot}$, referred to as the lower mass gap (LMG) and the upper mas gap (UMG), respectively. However, it is possible to form BHs in these gaps through merger of compact objects in dense clusters, e.g. the LMG and the UMG can be populated through binary neutron star- and BBH mergers, respectively. This implies that if binary mergers are observed in gravitational waves (GWs) with at least one mass gap object, then either clusters are effective in assembling binary mergers, or our single-star models have to be revised. Understanding how effective clusters are at populating both mass gaps have therefore major implications for both stellar- and GW astrophysics. In this paper we present a systematic study on how efficient stellar clusters are at populating both mass gaps through in-cluster GW mergers. For this, we derive a set of closed form relations for describing the evolution of compact object binaries undergoing dynamical interactions and GW merger inside their cluster. By considering both static and time evolving populations, we find in particular that globular clusters are clearly inefficient at populating the LMG in contrast to the UMG. We further describe how these results relate to the characteristic mass, time, and length scales associated with the problem.
\end{abstract}

Keywords: gravitational waves, neutron stars, black holes, black hole mass gaps, stellar dynamics

\section{INTRODUCTION}

Several gravitational wave $(\mathrm{GW})$ sources have now been observed by the LIGO and Virgo GW observatories, including both binary black holes (BBHs) (Abbott et al. 2016a,b,c, 2017a,b; Zackay et al. 2019; Venumadhav et al. 2019), and binary neutron stars (BNSs) (Abbott et al. 2017c). Their astrophysical origin is still unknown, but several formation channels have been suggested. Some of the recently proposed include: field binaries (Dominik et al. 2012, 2013, 2015; Kinugawa et al. 2014; Belczynski et al. 2016b,a; Silsbee \& Tremaine

Corresponding author: Johan Samsing

jsamsing@gmail.com
2017; Murguia-Berthier et al. 2017; Rodriguez \& Antonini 2018; Schrøder et al. 2018), dense stellar clusters (Portegies Zwart \& McMillan 2000; Banerjee et al. 2010; Tanikawa 2013; Bae et al. 2014; Rodriguez et al. 2015, 2016a,b,b; Askar et al. 2017; Park et al. 2017; Samsing 2018; Samsing \& D'Orazio 2018; Samsing et al. 2019a), active galactic nuclei (AGN) discs (Bartos et al. 2017; Stone et al. 2017; McKernan et al. 2017; Tagawa et al. 2019), galactic nuclei (GN) (O'Leary et al. 2009; Hong \& Lee 2015; VanLandingham et al. 2016; Antonini \& Rasio 2016; Stephan et al. 2016; Hoang et al. 2017; Hamers et al. 2018), very massive stellar mergers (Loeb 2016; Woosley 2016; Janiuk et al. 2017; D’Orazio \& Loeb 2017), and single-single GW captures of primordial black holes (Bird et al. 2016; Cholis et al. 2016; Sasaki et al. 2016; Carr et al. 2016). 
The question is, which of these proposed merger channels dominate the merger rate? Are several channels operating with a possible dependence on redshift? Or are the majority of GW sources formed through a still unknown mechanism? Several studies show that one can distinguish at least classes of channels apart, such as isolated binaries and dynamically induced mergers, by considering the observed distribution of merger masses (Zevin et al. 2017), the relative spin orientation of the merging objects (Rodriguez et al. 2016c), as well as the orbital eccentricity at some reference GW frequency (Gültekin et al. 2006; Samsing et al. 2014; Samsing \& Ramirez-Ruiz 2017; Samsing \& Ilan 2018; Samsing et al. 2018b; Samsing 2018; Samsing et al. 2018a; Samsing \& D'Orazio 2018; Rodriguez et al. 2018; Zevin et al. 2019; Samsing et al. 2019b,a). Other 'indirect' probes have also been suggested, such as stellar tidal disruptions (e.g. Samsing et al. 2019c; Lopez et al. 2019; Kremer et al. 2019a). In this picture, it is now largely believed that dynamically assembled mergers are likely to have mass rations near one (e.g. Rodriguez et al. 2018), random relative spin orientations (e.g. Rodriguez et al. 2016c), and a non-negligible fraction of mergers with measurable eccentricity in both LISA (Samsing \& D'Orazio 2018; Kremer et al. 2019b), DECIGO/Tian-Qin (e.g. Chen \& Amaro-Seoane 2017; Samsing et al. 2019a), and LIGO (Samsing 2018). This is in contrast to isolated binary mergers, that likely have correlated spins (e.g. Kalogera 2000), a bimodal distribution for the effective spin parameter (Zaldarriaga et al. 2018; Hotokezaka \& Piran 2017; Piran \& Piran 2020), larger mass ratios, and merge on orbits with eccentricities indistinguishable from $\approx 0$ near LISA and LIGO. This picture is rather clean when comparing mergers forming in highly dynamical systems, such as globular clusters (GCs) and GN, to completely isolated field binary mergers; however, it becomes less clean when considering e.g. the proposed sub-population of field binaries that undergo secular interactions with nearby single or binary objects (e.g. Naoz et al. 2013; Naoz 2016; Toonen et al. 2016; Antonini et al. 2017; Silsbee \& Tremaine 2017; Liu \& Lai 2018; Rodriguez \& Antonini 2018; Randall \& Xianyu 2018a; Antonini et al. 2018; Liu \& Lai 2019; Fragione \& Loeb 2019; Fragione \& Kocsis 2019; Hamers \& Thompson 2019; Safarzadeh et al. 2020). In this case, secular exchanges of especially angular momentum, can drive the binary to merge with random spin orientations (e.g. Liu \& Lai 2017), and notable eccentricity (e.g. Randall \& Xianyu 2018b; Liu et al. 2019; Fragione \& Kocsis 2020), which makes it more challenging to disentangle cluster mergers from field binary mergers.
An additional outcome that is somewhat unique to dynamically environments is the formation of so-called hierarchical mergers (e.g. O'Leary et al. 2016; Fishbach et al. 2017; Gerosa \& Berti 2017; Yang et al. 2019; Antonini et al. 2019; Gerosa \& Berti 2019; Samsing \& Ilan 2019; Rodriguez et al. 2019; Gerosa et al. 2020; Safarzadeh et al. 2020; Gayathri et al. 2020; Kimball et al. 2020; Doctor et al. 2020; Baibhav et al. 2020). The picture is here that compact objects (COs) that merge inside their cluster through e.g. single-single GW captures (e.g. Samsing et al. 2019a) or through chaotic fewbody interactions (e.g. Samsing et al. 2014; Zevin et al. 2019), will form a new population of 'second-generation' $(2 \mathrm{G})$ objects that are characterized by having a higher mass than the original 'first-generation' (1G) population, and a dimensional spin parameter around 0.7 (e.g. Berti et al. 2007). This $2 \mathrm{G}$ population can undergo further interactions leading to merger with other $1 \mathrm{G}$ or $2 \mathrm{G}$ objects, which then naturally will lead to an observable modified BBH mass spectrum, and spin distribution. This process can in principle also lead to 3G-, 4G-, ..., NG-populations, which naturally gives rise to unique observables. Looking for such hierarchical merger configurations has been proposed to be one way of probing the origin of GW mergers in very dense systems, such as GCs (Rodriguez et al. 2019), GN (Antonini \& Rasio 2016), and AGN disks (Yang et al. 2019). However, fine-tuned few-body configurations in the binary field population can in principle also create hierarchical mergers (e.g. Safarzadeh et al. 2020), but in this case its highly unlikely to go beyond $2 \mathrm{G}$. In any case, an observation of a hierarchical merger would strongly indicate that at least some GW sources are assembled as a result of few-body interactions.

Another interesting consequence of the hierarchical merger scenario is the possibility of populating the socalled lower mass gap (LMG) and upper mass gap (UMG), where the LMG is $\sim 3 M_{\odot}-5 M_{\odot}$ (Bailyn et al. 1998; Özel et al. 2010; Farr et al. 2011) and the UMG is marked by a lower limit of $\sim 45 M_{\odot}$ (Woosley 2017; Leung et al. 2019; Farmer et al. 2019). For example, the LMG might be populated through BNS collisions, where the UMG can be populated by BBH mergers. This makes it possible for dense clusters to produce GW sources with objects in either the LMG or the UMG. If 'Nature' is not able to form BHs through single star evolution in these mass gaps, then an observation of GW sources with a mass-gap object will give us insight into the fraction of mergers assembled in clusters, or at least dynamically. These mass-gaps not only play a key role in stellar-astrophysics, but introduce also a character- 
istic mass scale that can be used to e.g. constrain the cosmological parameters (Farr et al. 2019).

Several recent studies have discussed the possibility of populating the mass-gap in clusters (e.g. Rodriguez et al. 2019; Doctor et al. 2020; Baibhav et al. 2020). Currently, numerical studies suggest that BNS mergers are not likely to form in systems such as GCs (e.g. Ye et al. 2020). On the other hand, recent observations of the orbital parameters of galactic BNSs interestingly indicate that BNSs might actually form in clusters at rates several orders-of-magnitude higher than suggested by the numerical studies (e.g. Andrews \& Mandel 2019), which of course poses some interesting tension. Regarding BBHs, several studies have found that if the initial $\mathrm{BH}$ spins are low, then up to $\sim 10 \%$ of $\mathrm{BBH}$ mergers from GCs could be in the form of 1G-2G binaries, with a sub-fraction of these being in the UMG (Rodriguez et al. 2019). To find the observable contribution from such hierarchical mergers in upcoming and future GW data several numerical techniques and models are now under development (e.g. Doctor et al. 2020); however, common for the majority of these models is that they are not linked to any real physical model, they are instead just generic functional forms with a few fitting parameters. This kind of model independent approach might be useful to condense a huge stream of data into just a few fitting parameters, but gives a-priori no astrophysical insight into what systems that are likely and able to undergo hierarchical mergers and populating the mass-gaps.

In this paper we derive a set of fundamental relations describing how effective a dense cluster can grow a 2Gpopulation from a series of in-cluster GW mergers of $1 \mathrm{G}$ $1 \mathrm{G}$ binaries, as a function of characteristic mass, length, and time scales of the $1 \mathrm{G}$ objects and their cluster. The core of our calculations are based on the post-Newtonian $(\mathrm{PN})$ binary-single hardening model presented in Samsing (2018); Samsing \& D'Orazio (2018), where binaries are able to merge in-between or during their hard binary-single interactions. We use our derived expressions to make general statements about what clusters that are able to populate the LMG through BNS mergers, and the UMG through BBH mergers. Our model is fully analytical and our results are given in closed form expressions, and as a result, we are therefore only able to describe idealized clusters with constant density and velocity dispersion (for an extension of our model see e.g. Antonini \& Gieles 2020); however, our work serves as an important first step in connecting physical parameters with more general statements related to hierarchical mergers (see also recent work by Baibhav et al. 2020).
The paper is organized as follows. In Sec. 2 we introduce our dynamical cluster- and 3-body interaction model, and use it to derive results on how efficient a simple non-evolving binary and single cluster population is at producing in-cluster GW mergers. In Sec. 3 we extend our model to include a time dependent distribution of both singles and binaries, from which we derive a closed form solution to the upper limit on the number of $2 \mathrm{G}$-objects relative to $1 \mathrm{G}$-objects a given cluster can reach in a Hubble time. We further discuss these results in relation to populating the LMG and the UMG. We conclude our study in Sec. 4.

\section{FORMATION OF IN-CLUSTER MERGERS}

We consider a cluster with a population of COs (NSs or BHs), each with a mass $m$. These COs interact, and can through different dynamical pathways merge through the emission of GWs either inside or outside of their cluster (e.g. Samsing \& D'Orazio 2018; Rodriguez et al. 2018). The COs that merge inside the cluster give rise to a growing in-cluster population of $\mathrm{BHs}$ with a mass $\approx 2 m$, given that the kick velocity associated with asymmetric GW emission at merger is smaller than the cluster escape velocity (e.g. Gerosa \& Berti 2019). In this work we refer to the initial population of COs by ' 1 ' or 1 . generation $(1 \mathrm{G})$ objects, and the population of $\mathrm{BHs}$ that is formed through the collision of $1 \mathrm{G}-1 \mathrm{G}$ binaries by ' 2 ', or 2 . generation $(2 \mathrm{G})$. As described in the Introduction, the $2 \mathrm{G}$ population is able to populate both the lower $\left(3-5 M_{\odot}\right)$ and upper $\left(>45 M_{\odot}\right) \mathrm{BH}$ mass gaps that are believed to be associated with the initial $1 \mathrm{G}$ population. For example, it might be possible to populate the $3-5 M_{\odot} \mathrm{BH}$ mass-gab through the collision of BNSs.

Below we start by deriving and present a set of basic relations for describing the growth of $2 \mathrm{G}$ populations through in-cluster 1G-1G GW mergers. Throughout the paper we mainly illustrate results for our two fiducial mass cases; $m=1.4 M_{\odot}$ and $m=30 M_{\odot}$, which are in the relevant range for populating the LMG and the UMG, respectively.

\subsection{Cluster Model and 3-Body Dynamics}

In this work we study a model described by a cluster consisting of COs all with the same mass $m$. The cluster itself is assumed to have a constant number density of singles $n$, velocity dispersion $v_{d}$, and escape velocity $v_{e}=f_{e d} \times v_{d}$. Besides this single population, the cluster also harbors a population of $\mathrm{CO}$ binaries, that at early times consist of $1 \mathrm{G}-1 \mathrm{G}$ pairs, but at later times, through dynamical exchange interactions, can evolve to have pairs also including $2 \mathrm{G}$ objects. The binaries play 
a very important role, as these provide the main pathway for producing $2 \mathrm{G}$-objects as a result of binary-single interactions. In-cluster GW mergers can also form in other ways, such as through single-single GW captures (Samsing et al. 2019a), secular Kozai triples (Antonini et al. 2016), and binary-binary interactions (Zevin et al. 2019); however, these pathways are generally subdominant compared to the binary-single channel. Our main discussions will therefore mostly involve mergers from the interacting binary-single population. In the sections below we continue by describing the basics of our cluster model.

\subsubsection{Binary Hardening and Outcomes}

We assume that a given CO binary inside the cluster forms (dynamically) with a semi-major (SMA), $a$, equal to the hard-binary (HB) limit value (e.g. Heggie 1975; Aarseth \& Heggie 1976; Hut \& Bahcall 1983),

$$
a_{H B}=\frac{3 G}{2} \times \frac{m}{v_{d}^{2}},
$$

which is where the binary binding energy $\left(G m^{2} /(2 a)\right)$ equals the kinetic energy of the surrounding singles w.r.t. the binary $\left(m v_{d}^{2} / 3\right)$. After this, the binary undergoes scatterings with the surrounding singles, each of which leads to a decrease in the SMA of the binary from $a$ to $\delta a$. This corresponds to a change in $a$ of $-a(1-\delta)=-a \Delta$, where we have introduced $\Delta \equiv 1-\delta$ to shorten notations. In reality, the change per interaction in the binary binding energy $E_{b}$ follows approximately a power-law distribution $P\left(E_{b}\right) \propto E_{b}^{-\gamma}$ with $\gamma \sim 9 / 2$, depending on exactly how a strong binary-single interaction is defined (e.g. Heggie 1975; Stone \& Leigh 2019). In this work we do not use the full distribution, instead we assume that each interaction leads to a fixed fractional decrease $\delta$ in the SMA, that is equal to the average value found from the distribution $P\left(E_{b}\right) \propto E_{b}^{-\gamma}$. Using that $E_{b}=-G m^{2} /(2 a)$, and that $\delta \equiv\langle a\rangle / a_{0}$, where $a_{0}$ is the initial SMA and $\langle a\rangle$ is the average value of the resulting SMA, then $\delta$ is given by (see also Samsing 2018),

$$
\begin{aligned}
\delta & =(\gamma-1) \int_{0}^{1} \delta^{(\gamma-1)} d \delta=1-\gamma^{-1} \\
& =7 / 9, \quad(\gamma=9 / 2)
\end{aligned}
$$

The binary keeps undergoing these so-called 'hardening' interactions with the surrounding single population, until its SMA reaches one of the following three characteristic values: The first, denoted by $a_{e j}$, is the maximum SMA value for which the binary will get ejected out of the cluster if it undergoes a binary-single interaction. Note that this is a fixed value in our simple ' $\delta$-model'.
The second, denoted by $a_{G W}$, is the SMA for which the total integrated probability for the binary to merge at any given state from $a_{H B}$ to $a_{G W}$ equals one. The merging binary will of course have a decreasing SMA as it inspirals, but will during this time not interact with other objects. The third, denoted by $a_{t H}$, is the value it takes a Hubble time to reach through binary-single interactions alone from the initial value $a_{H B}$. The hierarchy of these three characteristic scales is set by $\left(v_{d}, n, m\right)$, and plays a key role in how to grow a $2 \mathrm{G} B \mathrm{BH}$ population inside the cluster through in-cluster mergers (see also Antonini \& Rasio 2016; Antonini \& Gieles 2020; Baibhav et al. 2020). For example, if $a_{e j}>a_{G W}$ then most binaries will get ejected and merge outside of the cluster, compared to if $a_{G W}>a_{e j}$ in which case all binaries will merge inside. If on the other hand $a_{t H}>\left\{a_{e j}, a_{G W}\right\}$ then the system will not be able to conclude even a single interaction sequence, and an effective accumulation of $2 \mathrm{G}$ mergers is therefore near impossible. As a result, the 'relevant' value for a given system is

$$
a_{m}=\max \left(\left\{a_{e j}, a_{G W}, a_{t H}\right\}\right),
$$

where the sub-script ' $m$ ' here refers to 'minimum', as this is the smallest value the SMA of the interacting binary can take. This $\delta$-model is further illustrated and described in Fig. 1.

Throughout the paper we refer to the process in which the system brings a binary from $a_{H B}$ to $a_{m}$ through binary-single interactions alone as one 'Interaction $\mathrm{Cy}$ cle' (IC). After a binary has completed its IC, then it generally happens that a new binary dynamically forms with a SMA $\sim a_{H B}$, after which the process repeats. This cycle of binary formation and hardening interactions is also often referred to as 'binary burning' (e.g. Kremer et al. 2020). We continue below by deriving $a_{e j}, a_{G W}$, and $a_{t H}$. We also refer the reader to (Antonini \& Rasio 2016; Antonini \& Gieles 2020; Baibhav et al. 2020) for complementary discussions on this.

\subsubsection{Derivation of Outcome Conditions}

For calculating the SMA at which the binary is ejected, $a_{e j}$, we first use that the energy released in one interaction between a single and a binary with SMA $a$ is given by $E_{b s}=(\Delta / \delta) \times E_{b}(a)$, where $E_{b}(a)$ is the internal energy of the binary before interaction (e.g. Samsing 2018). The energy $E_{b s}$ is 'released' in the three-body center-of-mass (COM), which in the Newtonian limit is conserved from before to after the interaction. From momentum conservation it then follows that the binary receives a velocity kick, defined at infinity in the COM, of $v_{b}^{2}=E_{b s} /(3 m)=(1 / 6)(\Delta / \delta) G m / a$. When $a$ is such that $v_{b}>v_{e}$ then the binary escapes the cluster. By 


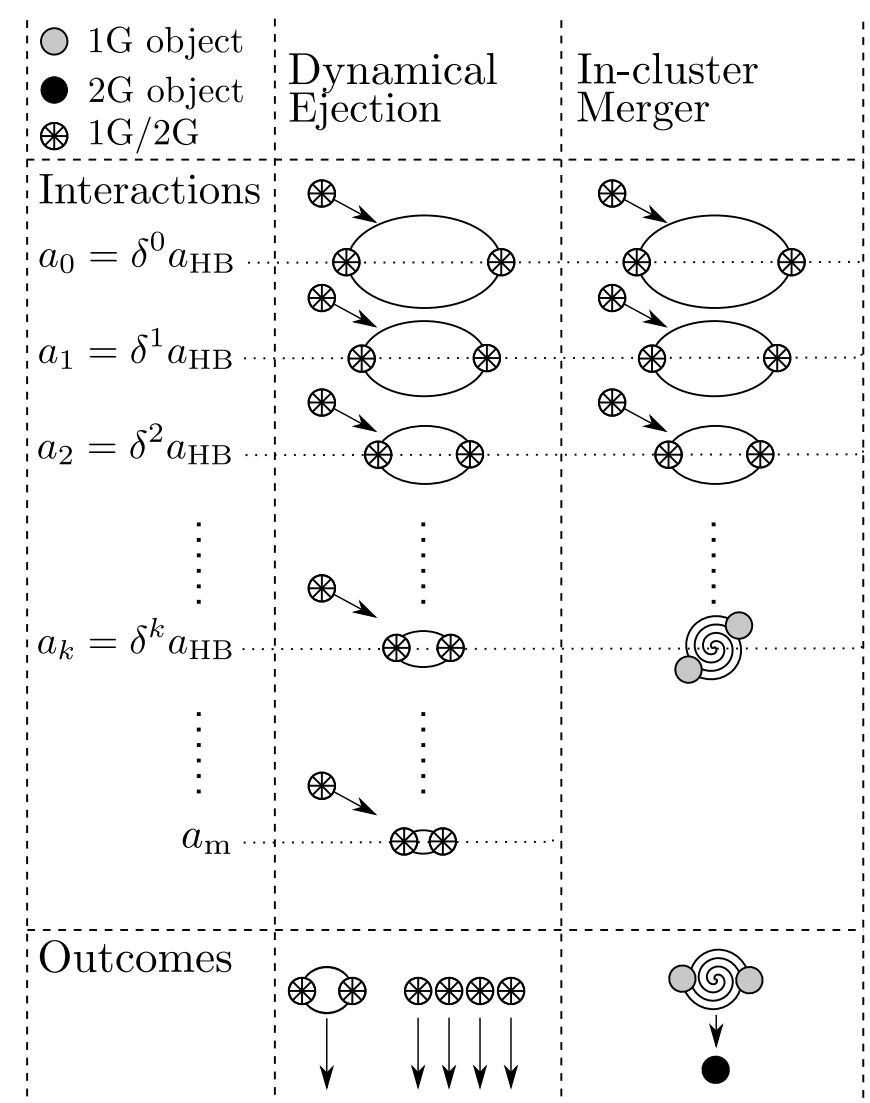

Figure 1. Illustration of our $\delta$-model described in Sec. 2.1. In this model we assume that all binaries dynamically form at the HB-limit inside the cluster, after which they undergo scatterings with the surrounding singles. Each scattering leads to a fixed decrease in the SMA from $a$ to $\delta a$, as shown in the left column (Interactions). This series of hardening interactions terminate at a characteristic SMA $a_{m}$, that either is where the binary mergers inside the cluster, is being ejected, or when the time passes $t_{H}$, as further described in Sec. 2.1.1. The middle and the right columns show the two scenarios where the binary is either ejected (Dynamical Ejection), or merges inside the cluster (In-cluster Merger), respectively. The outcome from each of these scenarios is shown in the bottom panel (Outcomes). As seen, the outcome from a dynamical ejection is 1 binary and $\sim 4$ singles, where for $1 \mathrm{G}-1 \mathrm{G}$ in-cluster mergers the outcome is per defintion a $2 \mathrm{G}$-object. When following the in-cluster population of $1 \mathrm{G}$ and $2 \mathrm{G}$ objects over time, the Dynamical Ejection outcome always acts as a 'sink term', where the $1 \mathrm{G}-1 \mathrm{G}$ In-cluster Merger outcome is the 'source term' for the $2 \mathrm{G}$ population, as further described in Sec. 3.1. Note that the grey and black circles refer to $1 \mathrm{G}$ - and $2 \mathrm{G}$-objects, respectively, where the diamond symbol denotes either of these two objects.

now defining $a_{e j} \equiv a\left(v_{b}=v_{e}\right)$ it then follows that,

$$
a_{e j} \approx \frac{G \Delta}{6 \delta f_{e d}^{2}} \times \frac{m}{v_{d}^{2}} .
$$

Note here that $a_{H B} / a_{e j}=9 f_{e d}^{2} \delta / \Delta=(63 / 2) f_{e d}^{2}$, where we have set $\delta=7 / 9$ in the last equality. A single bi- nary therefore has to decrease its SMA by 1-2 orders of magnitude through binary-single scatterings before a possible ejection can take place. As will be discussed and used later, several of the single objects interacting with the binary will also get ejected, as they likewise receive recoil kicks during the hardening process. As a result, for every single binary ejected there will also be $N_{s}^{e j}$ single objects ejected. This number $N_{s}^{e j}$ can be estimated by first comparing the SMA below which single ejections are possible, $a_{e j}^{s} \approx 2 G \Delta /\left(3 \delta f_{e d}^{2}\right) \times m / v_{d}^{2}$, where we have used $2 v_{b}=v_{s}$, with the binary ejection SMA $a_{e j}$ from Eq. (4). As seen, $a_{e j}^{s} / a_{e j}=4$. Now using that after $\Delta n$ binary-single interactions the binary SMA decreases by a factor $\delta^{\Delta n}$, it then follows that $N_{s}^{e j}=\ln (1 / 4) / \ln \delta \approx 5$, where we have used that one single object is ejected in each scattering for $a_{e j} \leq a \leq a_{e j}^{s}$. The number $N_{s}^{e j}$ is therefore a constant that does not depend on any properties of the system, as long all the interaction steps are 'available'. In this paper we use $N_{s}^{e j}=4$, as this value is slightly closer to what is found in numerical simulations; however, the exact value does not play a large role, the important point is that it takes a constant value.

For $a_{G W}$, we start by calculating the probability that a binary with SMA a merges before its next binarysingle interaction, denoted here by $p_{2}(a)$. For this we assume that the eccentricity distribution of the binary follows that of a thermal distribution, $P(e)=2 e$ (e.g. Heggie 1975). In addition, we use that the time inbetween binary-single interactions, $t_{b s}(a)$, is the inverse of the binary-single encounter rate, $t_{b s}(a) \approx\left(n \sigma_{b s} v_{d}\right)^{-1}$, where $\sigma_{b s} \propto m a / v_{d}^{2}$ is the HB binary-single interaction cross section (see e.g. Samsing et al. 2018b). Under these assumptions it directly follows that $p_{2}(a)=$ $\left(t_{b s}(a) / t_{G W}(a)\right)^{2 / 7}$, where $t_{G W}(a) \propto a^{4} / m^{3}$ is the GW inspiral life time corresponding to $e=0$ (e.g. Samsing 2018). This $p_{2}(a)$ is only the probability for merger during a single 'interaction step' $k$, where we here introduce the notation $a_{k}=a_{H B} \delta^{k}$. The total probability for a binary to merge in-between its binary-single interactions from $a_{H B}$ to $a_{m}$, denoted by $P_{2}\left(a_{m}\right)$, is therefore found by simply integrating from $k\left(a_{H B}\right)=0$ to $k\left(a_{m}\right)$. Using that $d a=-a \Delta d k$, the solution is found to be $P_{2}\left(a_{m}\right) \approx p_{2}\left(a_{m}\right) \times(7 /(10 \Delta))$ (e.g. Samsing 2018; Samsing et al. 2019c), which can be written out as,

$$
P_{2}\left(a_{m}\right) \approx A_{c}^{2 / 7} \times \frac{m^{4 / 7} v_{d}^{2 / 7}}{n^{2 / 7} a_{m}^{10 / 7}},
$$

where we have assumed that $p_{2}\left(a_{m}\right) \gg p_{2}\left(a_{H B}\right)$ and defined the constant $A_{c}=\left(7^{7 / 2} 85 G^{2}\right) /\left((10 \Delta)^{7 / 2} 9 \pi c^{5}\right)$. If we now set $P_{2}=1$ then the corresponding $a_{G W} \equiv$ 
$a\left(P_{2}=1\right)$ can now be isolated and gives,

$$
a_{G W}=A_{c}^{1 / 5} \times \frac{m^{2 / 5} v_{d}^{1 / 5}}{n^{1 / 5}} .
$$

As seen, this limit is surprisingly insensitive to the cluster parameters $v_{d}$ and $n$ (see also Antonini \& Rasio 2016)

The last characteristic SMA we consider is $a_{t H}$, which is the value for which it takes the binary a Hubble time to reach from $a=a_{H B}$ through binary-single interactions alone. For calculating this, we start with the time it takes the binary to undergo one interaction at interaction step ' $k$ ', which can be approximated as $t_{b s}\left(a_{k}\right) \approx\left(n \sigma_{b s}\left(a_{k}\right) v_{d}\right)^{-1}$ (see the above paragraph). The total time it takes to reach $a_{m}$, denoted by $\tau_{m}$, is found by integration $t_{b s}\left(a_{k}\right)$ from $k\left(a_{H B}\right)=0$ to $k\left(a_{m}\right)$. From this, one finds that $\tau_{m} \approx t_{b s}\left(a_{m}\right) / \Delta$, which also can be written as,

$$
\tau_{m} \approx(6 \pi G \Delta)^{-1} \times \frac{1}{a_{m}} \frac{v_{d}}{n m},
$$

where we have assumed that $a_{H B} \gg a_{m}$. Setting this expression for $\tau_{m}$ equal to $t_{H}$, and isolating the corresponding $a_{t H} \equiv a_{m}\left(\tau_{m}=t_{H}\right)$, one now finds,

$$
a_{t H} \approx \frac{(6 \pi G \Delta)^{-1}}{t_{H}} \times \frac{v_{d}}{n m}
$$

which relates to $a_{H B}$ as $a_{H B} / a_{t H} \approx\left(t_{H} / t_{b s}\left(a_{H B}\right)\right) \Delta$.

\subsection{Results}

Having derived analytical expressions for the three characteristic scales $a_{e j}, a_{G W}$, and $a_{t H}$ in Sec. 2.1.2 above, we are now in a position to start exploring what cluster systems that are likely to grow a population of $2 \mathrm{G}$ objects. In the sections below we study this by considering a few general relations and overview figures for a 'static' cluster population. In Sec. 3 we use these results to model 'time evolving' populations.

\subsubsection{Outcome Regions}

Fig. 2 shows $a_{m}=\max \left(\left\{a_{e j}, a_{G W}, a_{t H}\right\}\right)$ with colored regions (blue, red, grey) as a function of cluster velocity dispersion $v_{d}$ and number density $n$ for $m=30 M_{\odot}$ (top) and $m=1.4 M_{\odot}$ (bottom). The three regions are separated by green dashed lines, where the point at which all of the three regions meet, a point we refer to the 'break point' (BP), is highlighted with a green circular dot. How the green dashed lines depend on the parameters $v_{d}, n, m$, provide the key to understand what systems that are likely to produce a sizable population of $2 \mathrm{G}$ objects. Below we study this in more detail. Our
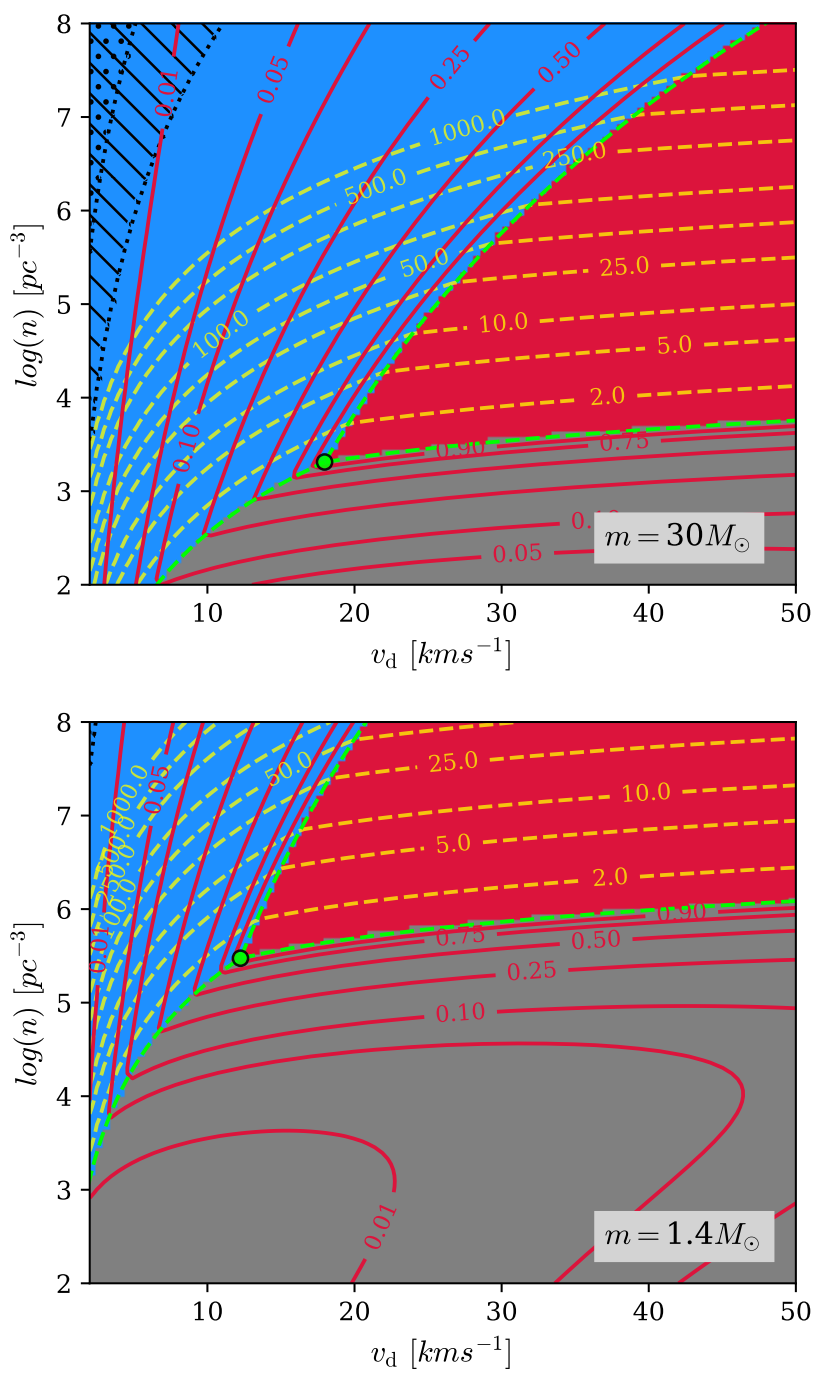

Figure 2. Results for a binary interacting with singles in a cluster described by a velocity dispersion $v_{d}=v_{e} / \sqrt{12}$, and number density $n$. The objects all have the same mass $m$, where $m=30 M_{\odot}(\mathrm{BH})$ and $m=1.4_{\odot}(\mathrm{NS})$ in the top plot and the bottom plot, respectively. The binary evolution is modeled using our $\delta$-model described in Sec. 2.1. In this model, the IC outcome of a given binary will fall into one of the following three general categories: (1) Blue region: The binary can get ejected through a binary-single interaction $\left(a_{m}=a_{e j}\right)$. (2) Red region: The binary will always merge inside the cluster before ejection is possible $\left(a_{m}=a_{G W}\right)$. (3) Grey region: The binary will not be able to finish a single IC within a Hubble time $\left(a_{m}=a_{t H}\right)$. The green dashed lines separating these outcome regions are discussed in Sec. 2.2.1. The red solid lines show the total probability for a binary to merge inside the cluster during one IC, $P_{M}=P_{2}+P_{3}$, where the orange dashed lines show the number of ICs a binary can undergo in Hubble time, $N_{c}\left(t_{H}\right)$. The hatched region shows where $P_{3}>P_{2}$, where the dotted region shows where $\Gamma_{s s}>\Gamma_{23}$ for $N_{b} / N_{s}=0.05$, as further described in Sec. 2.2.2. 
expressions are written out for $\delta=7 / 9$ if nothing else is stated.

We start by the line separating the two regions $a_{e j}$ (blue) and $a_{t H}$ (grey) to the left of the BP. By now setting $a_{e j}=a_{t H}$ and solving for the corresponding $n(e j, t H) \equiv n\left(a_{e j}=a_{t H}\right)$ one finds,

$$
n(e j, t H)=\left[\frac{63}{4 \pi} \frac{f_{e d}^{2}}{G^{2} t_{H}}\right] \times \frac{v_{d}^{3}}{m^{2}},
$$

where we used Eq. (4) and (8). The next line is the one separating the regions $a_{e j}$ (blue) and $a_{G W}$ (red) to the right of the BP. Following the same procedure as above, we first set $a_{e j}=a_{G W}$ and solve for the corresponding $n(e j, G W)$,

$$
n(e j, G W)=\left[\frac{1}{B_{c}} \frac{f_{e d}^{10}}{G^{3} c^{5}}\right] \times \frac{v_{d}^{11}}{m^{3}},
$$

where we have used Eq. (4) and (6), and introduced the constant $B_{c}=\left(9 \pi /\left(21^{5} 85\right)\right)(20 / 63)^{7 / 2}$. Finally, the line separating $a_{G W}$ (red) and $a_{t H}$ (grey) to the right of the $\mathrm{BP}$ is found from setting $a_{G W}=a_{t H}$, from which we find,

$$
n(G W, t H)=\left[\left(\frac{63}{4 \pi}\right)^{5} \frac{B_{c} c^{5}}{G^{7} t_{H}^{5}}\right]^{1 / 4} \times \frac{v_{d}}{m^{7 / 4}},
$$

where we have used Eq. (6) and (8). The associated coordinates of the BP, denoted by $\left(v_{d}(B P), n(B P)\right)$, can now be found from, e.g., setting $n(e j, t H)=n(e j, G W)$ from which follows,

$$
\begin{aligned}
& v_{d}(B P)=\left[\frac{63}{4 \pi} \frac{B_{c} G c^{5}}{f_{e d}^{8} t_{H}}\right]^{1 / 8} \times m^{1 / 8}, \\
& n(B P)=\left[\left(\frac{63}{4 \pi}\right)^{11} \frac{B_{c}^{3} c^{15}}{G^{13} f_{e d}^{8} t_{H}^{11}}\right]^{1 / 8} \times m^{-13 / 8} .
\end{aligned}
$$

As seen here, the $\mathrm{BP}$ coordinates $v_{d}(B P), n(B P)$ are $\propto m^{1 / 8}, m^{-13 / 8}$, respectively. Therefore, the location of the $\mathrm{BP}$ along the $v_{d}$-axis remains almost constant for reasonable changes in $m$, in contrast to the location along the $n$-axis, which can change by orders-ofmagnitude. As a result, for $1 \mathrm{G}$ objects in the mass range $1 M_{\odot} \lesssim m \lesssim 50 M_{\odot}$ the $\mathrm{BP}$ will always be around $10 \sim 20 \mathrm{kms}^{-1}$, which is slightly higher than the dispersion velocity of a typical GC. Since no configurations with $a_{m}=a_{G W}$ are possible for values of $v_{d}<v_{d}(B P)$ it then follows that GCs will in theory never be able produce binaries that only have the option of merging inside the cluster. The relevant value of $a_{m}$ for GCs is then either $a_{e j}$ or $a_{t H}$. This of course could also be used to argue why GCs have the properties they do, such as long time stability and no (visible) central massive BHs. Indeed, several studies have shown that velocity dispersion do act as central parameter for distinguishing e.g. GCs from NSCs with massive central BHs (e.g. Miller \& Davies 2012; Antonini \& Rasio 2016). If some GCs have massive BHs in the range of $10^{3}-10^{4} M_{\odot}$ in their center is still the focus of both observational (e.g. Kizlltan et al. 2017) and theoretical work (e.g. Gültekin et al. 2004; Giersz et al. 2015; Fragione \& Bromberg 2019; Hénault-Brunet et al. 2020), and could provide insight into the formation of the super-massive BHs seen in most galactic centre (e.g. Miller \& Davies 2012; Antonini et al. 2019). Another feature linked to the BP is that clusters with $n \gtrsim n(B P)$ will (nearly) always produce and process binaries that undergo at least one IC due to the relative weak dependence on $v_{d}$ for the $n(G W, t H)$-boundary. Regarding the dependence on $m$, one sees that the boundary quickly moves up for decreasing values of $m$, as $n(B P) \propto m^{-13 / 8}$. This makes it increasingly difficult for $1 \mathrm{G}$-objects with masses in the range $m \sim 1 M_{\odot}$ to undergo more than $1 \mathrm{IC}$ within a Hubble time for astrophysical cluster values compared to $m \sim 30 M_{\odot}$ 1G-objects, as clearly seen in Fig. 2 . Before we study this in greater detail, we proceed below by exploring to which degree 3-body mergers and singlesingle (S-S) GW captures contribute to the in-cluster merger rate.

\subsubsection{3-body Mergers and Single-Single GW Captures}

Before moving on to how efficient a population of binaries is at producing a $2 \mathrm{G}$ population, we here address the potential importance of including the in-cluster merger contribution from S-S GW captures and 3-body mergers. As described in Sec. 2.1, 'S-S GW captures' denote the process in which two initially unbound COs become bound through the emission of GWs (e.g. Samsing et al. 2019a), where a '3-body merger' refers to COs merging during a chaotic 3-body interaction (Samsing et al. 2014).

We start by analyzing the contribution from 3-body mergers. For this, we first estimate what part of the $\left(v_{d}, n\right)$-space the total integrated probability for producing a 3-body merger, $P_{3}$, is larger than the total probability for undergoing a 2-body merger, $P_{2}$. Following Samsing (2018), the probability for a binary-single interaction to produce a 3-body merger can be approximated by $p_{3}(a) \approx 2 \mathscr{N}\left(\mathscr{R}_{m} / a\right)^{5 / 7}$, where $\mathscr{N} \approx 20$ denotes the number of 'temporary binary states' the chaotic triple interaction on average assembles during one interaction, $\mathscr{R}_{m}$ is the Schwarzschild radius of a $\mathrm{BH}$ with mass $m$, and $a$ is the SMA of the initial target binary. The total probability for a 3-body merger to form during one IC 
can now be found from integrating $p_{3}(a)$ from $a=a_{H B}$ to $a=a_{m}$, in the same way as we did for finding $P_{2}$ in Sec. 2.1.2. Following this approach, one finds that $P_{3}\left(a_{m}\right) \approx p_{3}\left(a_{m}\right) \times(7 /(5 \Delta))$, which can be written out in the following way,

$$
P_{3}\left(a_{m}\right) \approx\left[\frac{2^{12 / 7} 7}{5 \Delta} \frac{\mathscr{N} G^{5 / 7}}{c^{10 / 7}}\right] \times m^{5 / 7} a_{m}^{-5 / 7}
$$

where we have assumed that $p_{3}\left(a_{m}\right) \gg p_{3}\left(a_{H B}\right)$ (Note here that we calculate these merger probabilities separately, i.e., we do not take into account the potential interplay between merger channels, including the S-S GW capture channel). From this we see that $P_{3}\left(a_{m}\right) / P_{2}\left(a_{m}\right) \propto\left(n / v_{d}\right)^{2 / 7}$. This indicates that 3 body mergers will provide the greatest contribution relative to the 2-body mergers at high $n$ and low $v_{d}$, which is the regime where $a_{m}=a_{e j}$, as seen on Fig. 2. We therefore need to evaluate and compare $P_{2}$ and $P_{3}$ for $a_{m}=a_{e j}$. Using Eq. (5), Eq. (13), and Eq. (4), this first lead us to,

$$
P_{2}\left(a_{e j}\right) \approx\left[\frac{1}{B_{c}} \frac{f_{e d}^{10}}{G^{3} c^{5}}\right]^{2 / 7} \times \frac{v_{d}^{22 / 7}}{m^{6 / 7} n^{2 / 7}}
$$

and

$$
P_{3}\left(a_{e j}\right) \approx\left[\frac{42^{5 / 7}}{(5 / 63)} \frac{\mathscr{N} f_{e d}^{10 / 7}}{c^{10 / 7}}\right] \times v_{d}^{10 / 7} .
$$

Now setting these two expressions equal to each other one finds,

$$
n\left(P_{2}, P_{3}\right) \approx\left[\frac{(5 / 63)^{7 / 2} f_{e d}^{5}}{42^{5 / 2} \mathscr{N}^{7 / 2} B_{c} G^{3}}\right] \times \frac{v_{d}^{6}}{m^{3}},
$$

where $n\left(P_{2}, P_{3}\right)$ therefore represents the boundary in the $a_{m}=a_{e j}$ region for which $P_{2}=P_{3}$. This boundary is shown in Fig. 2 with the dotted line that encloses the black solid line hatched area. In this area $P_{3}>P_{2}$. As seen, for most systems, especially the one with relative low mass $m$ and moderate density $n, 3$-body mergers will not dominate the total in-cluster merger probability. We will therefore in our analytical models throughout this paper omit this contribution for simplicity and clarity.

We now move on to the S-S GW capture population. For this its more easy to compare merger rates, $\Gamma$, than probabilities. In this case, the total rate of S-S GW capture mergers from a simple ' $n \sigma v$ ' estimate is given by (Samsing et al. 2019a),

$$
\Gamma_{s s} \approx\left[\frac{4 \pi G^{2}}{c^{10 / 7}}\left(\frac{85 \pi}{24 \sqrt{2}}\right)^{2 / 7}\right] \times \frac{N_{s} m^{2}}{v_{d}^{18 / 7}},
$$

where $N_{s}$ is the total number of single BHs. Note that we have here assumed that all the single $\mathrm{BHs}, N_{s}$, are distributed uniformly according to our model of a constant $v_{d}, n$; however, in reality, the single $\mathrm{BHs}$ naturally distribute according to some density and velocity profile. As a result, the real GW capture rate is generally smaller than the one presented in the above Eq. (17), as further discussed in Samsing et al. (2019a). Regarding the merger rate from our considered binary-single interactions, one finds that this can be approximated by,

$$
\Gamma_{23} \approx \frac{N_{b}\left(P_{2}\left(a_{m}\right)+P_{3}\left(a_{m}\right)\right)}{\tau_{m}},
$$

where $P_{2}+P_{3} \leq 1$ is the total number (probability) of 2-body and 3-body mergers forming during $1 \mathrm{IC}, \tau_{m}$ is the time it takes for undergoing 1 IC (see Eq. (7)), and $N_{b}$ is the number of CO binaries in the cluster that contributes to the merger rate. We have here included the 3-body mergers, as it turns out that the S-S GW captures only significantly contribute for low $v_{d}$ and high $n$, exactly where the 3 -body mergers also contribute. This is seen in Fig. 2, where the black dotted line inclosing the black dotted area is where $\Gamma_{s s}=\Gamma_{23}$ for binary fraction $N_{b} / N_{s}=0.05$. The S-S GW captures are therefore not expected to provide a significant contributing in the regions we are interested in.

To conclude, we have here shown and argued that neither the 3-body mergers nor the S-S GW capture mergers contribute significantly to the in-cluster merger rate. We therefore only consider the 2-body merger contribution in the rest of this paper.

\subsubsection{Interaction Cycles and In-cluster Mergers}

The number of in-cluster GW mergers that can be produced over a Hubble time per 1G-1G binary, here denoted by $N_{M}^{\prime}\left(t_{H}\right)$, serves as an approximate measure for how efficient a given cluster is at growing a $2 \mathrm{G}$ population. At this stage we approximate this number by the following product,

$$
N_{M}^{\prime}\left(t_{H}\right) \approx N_{c}\left(t_{H}\right) \times P_{M},
$$

where $N_{c}\left(t_{H}\right)=t_{H} / \tau_{m}$ is the number of ICs a cluster can run through in a Hubble time, i.e. the number of binaries the cluster can process in time $t_{H}$, and $P_{M}$ is the probability for an in-cluster GW merger to form during one IC. In Fig. 2 is shown with orange dashed lines and red solid lines the contours of $N_{c}\left(t_{H}\right)$ and $P_{M}$, respectively, where for $P_{M}$ we have here included the probability for 3-body mergers, i.e. $P_{M}=P_{2}\left(a_{m}\right)+$ $P_{3}\left(a_{m}\right)$. In short, our procedure for estimating $N_{c}\left(t_{H}\right)$ and $P_{M}$ at a given point $\left(v_{d}, n\right)$, is first to calculate $a_{m}$ from Eq. (3), after which we use Eq. (7) to find $N_{c}\left(t_{H}\right)=t_{H} / \tau_{m}$, and Eq. (5) and Eq. (13) to find $P_{M}=P_{2}\left(a_{m}\right)+P_{3}\left(a_{m}\right)$. 

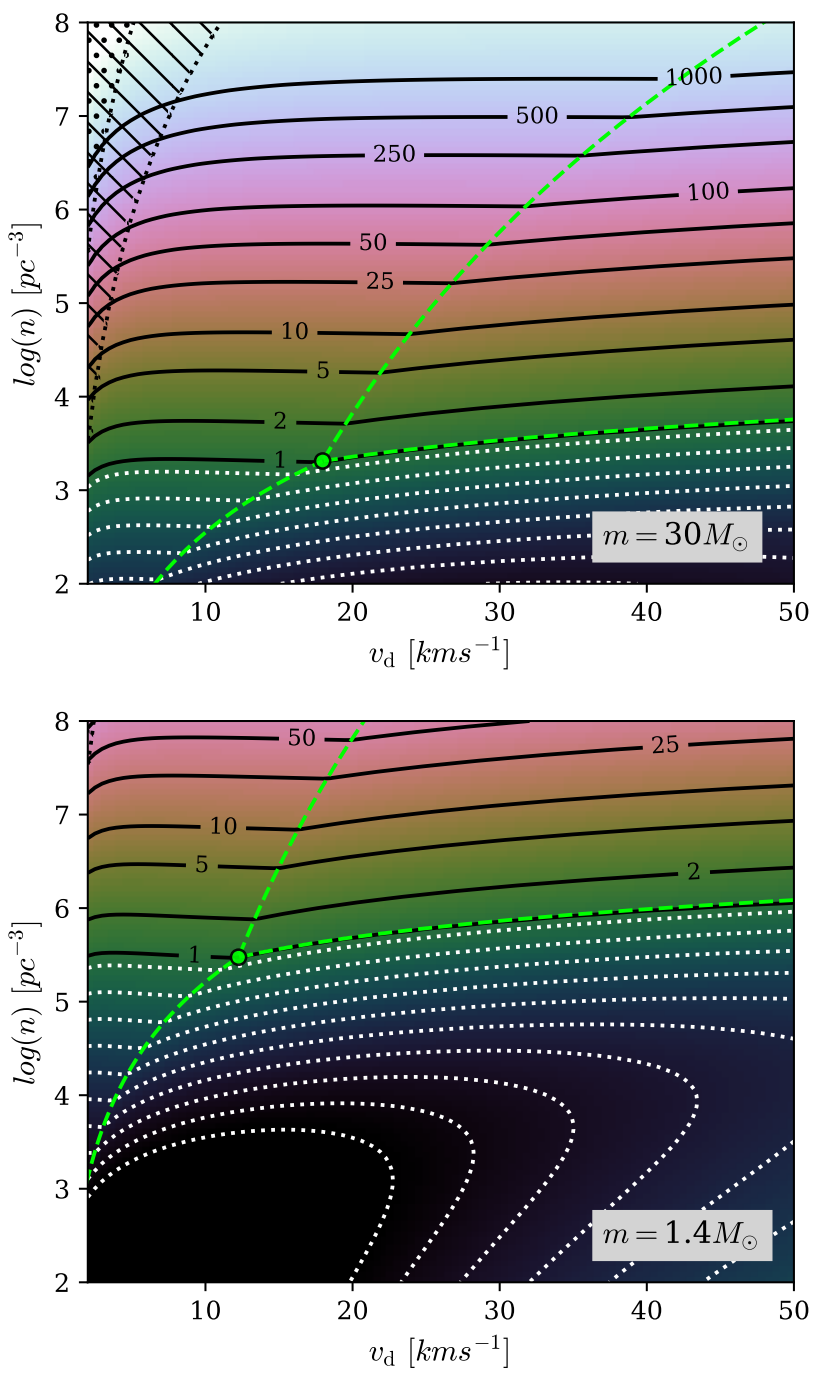

Figure 3. Similar to Fig. 2, but here the black solid lines show the corresponding number of in-cluster GW mergers that form in a Hubble time per binary, $N_{M}^{\prime}\left(t_{H}\right)$. The white dotted contour lines show where $N_{M}^{\prime}\left(t_{H}\right)<1$, and highlights therefore the area for which the system is not effective in growing a $2 \mathrm{G}$ population. For this figure we have assumed that $N_{M}^{\prime}\left(t_{H}\right) \approx N_{c}\left(t_{H}\right) \times P_{M}$, where $N_{c}\left(t_{H}\right)$ is the number of ICs a binary can undergo in a Hubble time, and $P_{M}=P_{2}+P_{3}$ is the probability for a binary to undergo an in-cluster GW merger during one IC. These two quantities are also shown separately in Fig. 2. As seen in the figure above, the number $N_{M}^{\prime}\left(t_{H}\right)$ is almost independent of $v_{d}$, but highly sensitive to especially $m$.

As seen in Fig. 2, for decreasing values of $v_{d}$ the probability $P_{M}$ decreases, which also follows from Eq. (14) where $P_{2} \propto v_{d}^{22 / 7}$, in contrast to the number of ICs, $N_{c}\left(t_{H}\right)$, that instead increases. Therefore, one can easily have a cluster with binaries where burning is efficient, i.e. where $N_{c}\left(t_{H}\right) \gg 1$, but at the same time with a probability for merging during individual ICs is low, i.e. with $P_{M} \ll 1$, and vice versa. How these two quantities 'balance out' is clear in Fig. 3, which shows in black solid lines $N_{M}^{\prime}\left(t_{H}\right)$ from Eq. (19). Surprisingly, the large changes in both $P_{M}$ and $N_{c}\left(t_{H}\right)$ as $v_{d}$ is varied almost cancel out, and $N_{M}^{\prime}\left(t_{H}\right)$ is as a result almost flat across $v_{d}$. To study this behavior further, we can write out $N_{M}^{\prime}\left(t_{H}\right)$ in the region relevant for GC systems where $P_{2} \gg P_{3}$ and $a_{m}=a_{e j}$ using Eq. (19), Eq. (7) evaluated at $a_{m}=a_{e j}$, and the expression for $P_{2}\left(a_{e j}\right)$ given by Eq. (14), from which one finds,

$$
\begin{aligned}
N_{M}^{\prime}\left(t_{H}\right) & \approx t_{H}\left[\left(\frac{4 \pi}{63}\right)^{7 / 2} \frac{G^{4} f_{e d}^{3}}{B_{c} c^{5}}\right]^{2 / 7} \times n^{5 / 7} m^{8 / 7} v_{d}^{1 / 7} \\
& \approx 0.5\left(\frac{n}{10^{5} p c^{-3}}\right)^{5 / 7}\left(\frac{m}{1.4 M_{\odot}}\right)^{8 / 7}\left(\frac{v_{d}}{10 k m s^{-1}}\right)^{1 / 7}
\end{aligned}
$$

where in the last line we have inserted values relevant for NS-NS mergers. This confirms the results we see in Fig. 3, namely that $N_{M}^{\prime}\left(t_{H}\right)$ only depends weakly on $v_{d}$ as $N_{M}^{\prime}\left(t_{H}\right) \propto v_{d}^{1 / 7}$. As a result, all systems with $n \gtrsim n(B P)$ will to leading order have $N_{M}^{\prime}\left(t_{H}\right) \gtrsim 1$. From this follows that if the number of $\mathrm{CO}$ binaries is constant in time at a value $N_{b}$, then the number of incluster mergers for $n \gtrsim n(B P)$ will be $\gtrsim N_{b}$ after a Hubble time. For example, for our $m=30 M_{\odot}$ case shown in the upper panel of Fig. 3, the number of incluster mergers over a Hubble time per binary is of order 10 for $\log n \approx 4 \sim 5 p c^{-3}$. If the number of BBHs in the cluster at any given time is a few, say $\sim 5$, then our model predicts that the total number of in-cluster mergers forming over a Hubble time is $\sim 5 \times 10=50$. Although this number of course fluctuates from cluster to cluster, we note that this number is consistent with what is found using numerical simulations (see Rodriguez et al. (2019), where 48 in-cluster mergers were reported for their example in Sec. IV.A). More generally, $N_{M}^{\prime}\left(t_{H}\right)$ provides an upper limit on the number of available $2 \mathrm{G}$ objects after a Hubble time produced per cluster binary, as only a small fraction of the in-cluster mergers, i.e. $2 \mathrm{G}$ objects, are actually retained by the cluster Rodriguez et al. (2019). The remaining are either kicked out immediately as a result of GW kicks, or later dynamically through e.g. a binary-single interaction. Considering the $m=1.4 M_{\odot}$ case, we see both from Eq. (21) and Fig. 3 that $N_{M}^{\prime}\left(t_{H}\right)$ is only $\gtrsim 1$ for $\log n \gtrsim 5-6 p c^{-3}$, which is a very high density threshold for astrophysical standards. This provides a clear hint that clusters hosting only NS-NS binaries are not likely to be effective in turning its population into a sizable 
$2 \mathrm{G}$ population, i.e. in populating the $\mathrm{LMG}$, unless the binary fraction initially is relatively high.

Lastly, in relation to the probability of observing a possible 2G-population from a cluster, what matters is not only the number of $2 \mathrm{G}$-objects produced, but also how many of these that are present in the cluster compared to the number of remaining $1 \mathrm{G}$ objects. As described back in Sec. 2.1.2, a single IC will on average give rise to $N_{e j}^{s}+2 \sim 6$ ejected 1G-objects (if in-cluster mergers and $2 \mathrm{G}$ objects are ignored), which naturally leads to a gradual reduction of this population over time. In our model considered so far, the number of in-cluster GW mergers relative to the number of (remaining) 1Gobjects after time $t$ is therefore approximately,

$$
\begin{aligned}
\frac{N_{M}^{\prime}(t)}{N_{1}(t)} & \approx \frac{N_{b} N_{M}^{\prime}(t)}{N_{1}(0)-N_{b} N_{c}\left(N_{e j}^{s}+2\right)} \\
& \approx \frac{N_{M}^{\prime}(t)}{f_{b}^{-1}(0)-N_{e j}^{\prime}(t)}
\end{aligned}
$$

where we have assumed that $N_{b}$ remains constant, $N_{1}(0)$ denotes the initial number of $1 G$-objects, $f_{b}(0)=$ $N_{b} / N_{1}(0)$, and $N_{e j}^{\prime}(t)$ denotes the total number of $1 G$ objects ejected after time $t$ per binary. We will explore this ratio and others in the sections below.

\section{POPULATING THE BLACK HOLE MASS GAPS: TIME EVOLVING CLUSTER MODEL}

In this section we develop a simple time dependent cluster model to study the evolution of both $1 \mathrm{G}$ and $2 \mathrm{G}$ objects as a function of time. As further described in the following sections, in this model we take into account both binary and single dynamical ejections, and in particular the growth of $2 \mathrm{G}$ objects as a result of incluster 1G-1G mergers. We (still) assume the cluster is described by a fixed set $v_{d}, n$, and all objects have the same mass $m$, which of course is a simplification of a real cluster. This in turn however enables us to put forward simple, general, and informative statements, based solely on characteristic mass, length, and time scales.

In the first section below we derive a set of evolution equations for $N_{1}$ and $N_{2}$, where $N_{i}$ here denotes the number of objects of type ' $i$ '. In Sec. 3.2 we solve these equations from which we put upper limits on the ratio $N_{2} / N_{1}$, illustrated for $m=1.4 M_{\odot}(2 \mathrm{G}$ in the $\mathrm{LMG})$ and $m=30 M_{\odot}(2 \mathrm{G}$ in the $\mathrm{UMG})$, for a grid of cluster systems described by $v_{d}, n$.

\subsection{Evolution Equations}

We consider a cluster described by a constant $v_{d}, n$ that initially has a population of $N_{1}(0) 1 \mathrm{G}$ objects all with equal mass $m$. In this cluster there is a (timedependent) population of binaries that interact with the
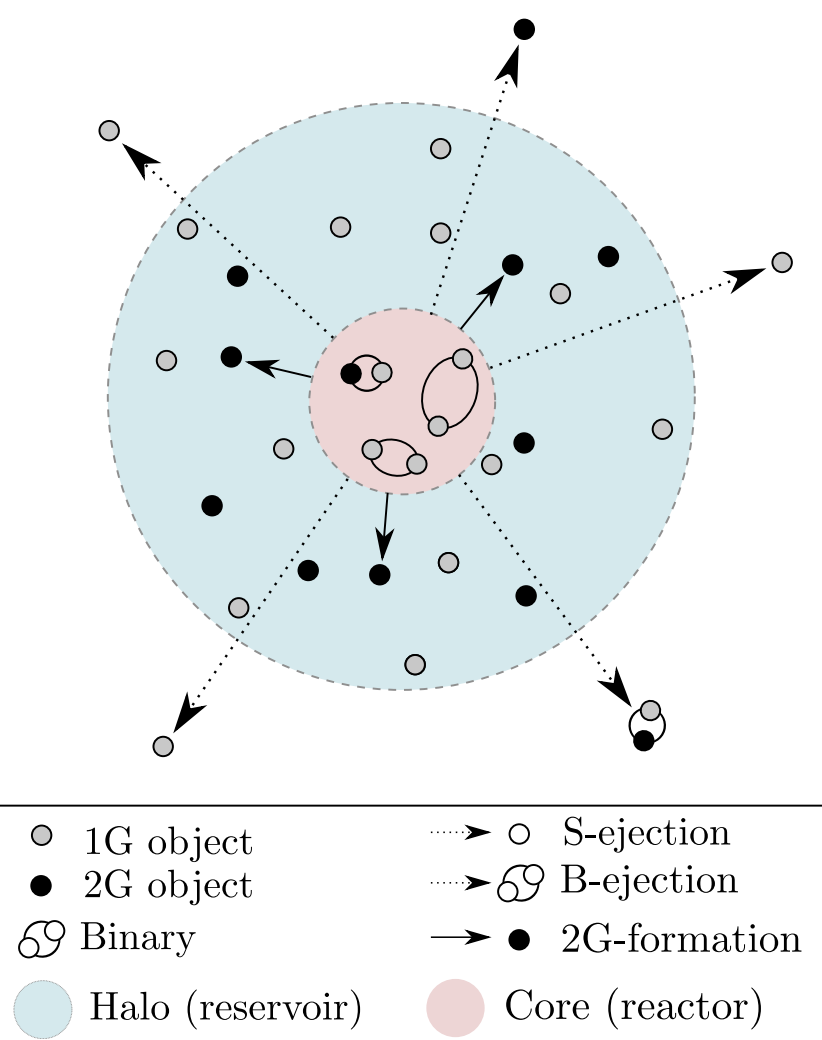

Figure 4. Illustration of the cluster model we use to study the formation of $2 \mathrm{G}$-objects (black dots) through successive in-cluster GW mergers of 1G-objects (grey dots). The cluster is composed of two parts, an inner core (pink region) and an outer halo (blue region). Inside the core there is $N_{b}$ binaries that interact with the flow of objects coming in from the outer halo. The resulting binary-single interactions are modeled using our $\delta$-model described in Sec. 2.1, which leads to both dynamical ejections of binaries (B-ejection) and singles (S-ejection), and the production of $2 \mathrm{G}$-objects ( $2 G$ formation) through in-cluster $\mathrm{GW}$ mergers of $1 \mathrm{G}-1 \mathrm{G}$ binaries. The number of $2 \mathrm{G}$-objects compared to the number of $1 \mathrm{G}$-objects, i.e. $N_{2} / N_{1}$, after a Hubble time provides a rough estimate for how likely it is to observe a GW source from a binary merger that includes at least one $2 \mathrm{G}$-object. We study the evolution of $N_{2} / N_{1}$ in Sec. 3, and comment on the implied possibility for populating the UMG and the LMG in Sec. 3.2.2.

surrounding single population, which give rise to dynamical ejections, exchanges, and in-cluster mergers. The absolute and relative number of $N_{1}(1 \mathrm{G})$ and $N_{2}(2 \mathrm{G})$ objects therefore changes over time through various dynamical mechanisms, that all depend on $v_{d}, n, m$. This configuration is described and illustrated in Fig. 4. The question is, for what initial conditions of $v_{d}, n, m$ is the system able to produce a sizable population of $2 \mathrm{G}$ objects after a Hubble time? To answer this question, we start by writing out the following set of differential equations that we take to represent the evolution of $N_{1}$ and 
$N_{2}$,

$$
\begin{aligned}
\dot{N}_{1}= & -\left(\dot{N}_{1}^{e j}+\dot{N}_{21}^{e j}+2 \dot{N}_{11}^{e j}\right)-\left(\dot{N}_{21}^{M}+2 \dot{N}_{11}^{M}\right) \\
\dot{N}_{2}= & -\left(\dot{N}_{2}^{e j}+\dot{N}_{21}^{e j}+2 \dot{N}_{22}^{e j}\right)-\left(\dot{N}_{21}^{M}+2 \dot{N}_{22}^{M}\right) \\
& +\left(R_{11}^{M} \dot{N}_{11}^{M}\right),
\end{aligned}
$$

where $N_{i}^{e j}$ is the number of objects of type ' $i$ ' (1G or $2 \mathrm{G}$ ) that are ejected as singles, $N_{i j}^{e j}$ is the number of ejected binaries consisting of object types ' $\{i j\}$ ', $N_{i j}^{M}$ is the number of $\{i j\}$-binaries merging in-side the cluster, and $R_{11}^{M}$ is the retention fraction of $1 \mathrm{G}-1 \mathrm{G}$ mergers. As seen, both 'ejections' (single and binary) and 'in-cluster mergers' all act as 'sink terms', except as for the term $\propto N_{11}^{M}$ that serves as the $2 \mathrm{G}$ 'source term'. As we are studying the process of growing a $2 \mathrm{G}$ population through in-cluster GW mergers during successive ICs, we restrict our self in the following to describe systems that are able to undergo $N_{c} \gg 1$. Therefore, instead of evolving the above equations over e.g. individual interaction steps ' $k$ ', or time, we evolve them over the number of ICs, $N_{c}$. The 'dot' over each $N$ refers therefore to the change per IC.

The relevant terms for writing out our evolution equations from above can be written as,

$$
\begin{aligned}
& \dot{N}_{i}^{e j} \approx\left[N_{b} \bar{P}_{M}\right] p_{i}^{e j} N_{s}^{e j}, \\
& \dot{N}_{i j}^{e j} \approx\left[N_{b} \bar{P}_{M}\right] p_{i j}^{e j}, \\
& \dot{N}_{i j}^{M} \approx\left[N_{b} P_{M}\right] p_{i j}^{b},
\end{aligned}
$$

where $N_{b}$ is the number of binaries, $P_{M}\left(\bar{P}_{M}=1-P_{M}\right)$ is the integrated probability that a given binary does (not) merge during a single IC, $p_{i}^{e j}$ is the probability that object ' $i$ ' is ejected after a binary-single interaction, $N_{s}^{e j}$ is the total number of singles per binary ejected during one IC, $p_{i j}^{e j}$ is the probability that binary-' $\{i j\}$ ' is ejected after a binary-single interaction, and $p_{i j}^{b}$ is the probability that $\{i j\}$ is in a binary at a random hardening step ' $k$ '. These terms can be further expanded as,

$$
\begin{array}{llll}
p_{2}^{e j} & \approx p_{2}^{i} p_{112}^{e s}[1+B], & & p_{1}^{e j} \approx 1-p_{2}^{e j} \\
p_{21}^{e j} \approx p_{2}^{i} p_{211}^{e s}[1+B], & & p_{11}^{e j} \approx 1-p_{21}^{e j} \\
p_{21}^{b} \approx p_{2}^{i} B, & & p_{11}^{b} \approx 1-p_{21}^{b} \\
p_{211}^{e s} \approx 2 w / 3, & & p_{112}^{e s} \approx 1-p_{211}^{e s} \\
p_{2}^{i} \approx N_{2} F /\left(N_{1}+N_{2}\right), & p_{1}^{i} \approx 1-p_{2}^{i} .
\end{array}
$$

where $p_{2}^{i}$ is the probability that object type ' 2 ' (2G) is the incoming single object in a binary-single interaction at hardening step ' $k$ ', $p_{i j k}^{e s}$ is the probability that a given binary-single interaction results in an endstate where $\{i j\}$ is a binary and ' $k$ ' leaves as single, and $B=2 F w /(3-2 w)$. The factor $F$ is introduced to quantify the probability 'enhancement' of a 2G-object to interact with a binary compared to a $1 \mathrm{G}$-object. For example, the enhancement factor from standard gravitational focusing of having a 2G-object to interact with a binary compared to a $1 \mathrm{G}$-object is $F=(1+1+2) /(1+1+1)=4 / 3$. Similarly, $w$ describes the 'enhanced probability' that the outcome of a binary-single interaction involving a $2 \mathrm{G}-$ object is $\{121\}$, i.e. where ' $\{12\}$ ' is a binary and ' 1 ' is ejected as single. For this set of equations we have made four central assumptions: (1) All binary-single interactions involving objects $\{i j k\}$ have the same outcome distributions irrespective of the initial configuration. (2) The probability to have interactions with $>12$ G-object is $=0$, which follows from our considered limit of $N_{2} \ll N_{1}$. (3) Dynamical single and binary ejections associated with a given interacting binary are only $>0$ if the binary in question does not merge before concluding its IC. (4) All interactions and ICs follow our ' $\delta$-model' illustrated in Fig. 1. Now using these equations we can rewrite our evolution equations given by Eq. (24) as follows,

$\dot{N}_{1}=N_{b} \times\left[+p_{2}^{i}\left(A-P_{M}(A-B)\right)-\left(N_{t}^{e j}-P_{M} N_{s}^{e j}\right)\right]$
$\dot{N}_{2}=N_{b} \times\left[-p_{2}^{i}\left(A-P_{M}(A-B)\right)+\left(p_{11}^{b} P_{M} R_{11}^{M}\right)\right]$,

where $N_{t}^{e j}=2+N_{s}^{e j}$ is here the total number of ejected objects over $1 \mathrm{IC}$, and $A=[1+B]\left(p_{112}^{e s} N_{s}^{e j}+p_{211}^{e s}\right)$.

To summarize, our presented evolution equations given Eq. (24) are completely general, and shows simply what characteristic sink and source terms that are relevant for our problem. Other terms, such as strong binary-binary interactions (Zevin et al. 2019), and weak few-body scatterings (Hamers \& Samsing 2019a; Samsing et al. 2019b; Hamers \& Samsing 2019b, 2020), or more general mass-ratio dependent terms and corresponding GW kick prescriptions can be included, but this is beyond this paper. The resulting terms shown in Eq. (33) follow directly from simple combinatorics, and are constructed by calculating the (time dependent) probability for $1 \mathrm{G}$ - and $2 \mathrm{G}$-objects to interact and exchange into the interactions states shown in Fig. 1, folded with the probability for dynamical ejections and in-cluster mergers during each IC. In the following sections we consider solutions to this coupled set of equations, from which we especially find a closed form solution to the upper limit on $N_{2} / N_{1}$ as a function of time.

\subsection{Results}

In the first section below, we study the evolution of $N_{1}$ and $N_{2}$ for two different cluster models, denoted $c A$ 
and $c B$, using the general set of evolution equations presented in the above Sec. 3.1. In the second section, we use these results to study the upper limit on the ratio $N_{2} / N_{1}$ evaluated at present day, i.e. at $t=t_{H}$, for a grid of $v_{d}, n$ cluster systems.

\subsubsection{Time-Evolving Populations}

We study the evolution of $N_{1}$ and $N_{2}$ using Eq. (33) for two distinct cases, $c A$ and $c B$. These two cases are described in the following.

Case ' $c A$ ': In this case we assume the weight factors $F=1$ and $w=1$, i.e., we keep track of the growing population of $2 \mathrm{G}$-objects, but assume that in all dynamical aspects a $2 \mathrm{G}$ object is indistinguishable from a $1 \mathrm{G}$ object. We are therefore able to explore the effect from pure 'combinatorics' arising from the growing population of $2 \mathrm{G}$-objects that are free to exchange, merge, and being ejected in the same way as the $1 \mathrm{G}$ objects. Using Eq. (33) with $F=1, w=1$ the evolution equations are in this case given by,

$$
\begin{aligned}
& \dot{N}_{1} / N_{b} \approx-p_{1}^{i}\left(N_{t}^{e j}-P_{M} N_{s}^{e j}\right) \\
& \dot{N}_{2} / N_{b} \approx-p_{2}^{i}\left(N_{t}^{e j}-P_{M} N_{s}^{e j}\right)+P_{M} R_{11}^{M}\left(1-2 p_{2}^{i}\right),
\end{aligned}
$$

where we have used that under these assumptions $A=$ $N_{t}^{e j}$ and $A-B=N_{s}^{e j}$. In this case the number of $2 \mathrm{G}$ objects compared to $1 \mathrm{G}$-objects present in the cluster after a Hubble time represents approximately a lower limit, as in 'reality' a higher number of $2 \mathrm{G}$-objects will be left in the cluster due to their higher mass (e.g. Sigurdsson \& Phinney 1993).

Case ' $c B$ ': In this case we assume that $p_{2}^{i}=0$ and $R_{11}^{M}=1$, i.e. that the $2 \mathrm{G}$-objects are not participating in any interactions, and that the $1 \mathrm{G}$-objects as a result dynamically evolve through interactions, merger, and ejections completely independent of the $2 \mathrm{G}$-objects. As a result, the number of $2 \mathrm{G}$-objects we here find after time $t_{H}$ represents the highest number possible, and the $1 \mathrm{G}$-population will also decrease to its lowest possible value. This case therefore represents the upper limit on how many $2 \mathrm{G}$-objects one can keep in a cluster after time $t_{H}$ compared to the 1G-population. The evolution equations are in this case given by Eq. (33) with $p_{2}^{i}=0$ and $R_{11}^{M}=1$,

$$
\begin{aligned}
& \dot{N}_{1} / N_{b} \approx-\left(N_{t}^{e j}-P_{M} N_{s}^{e j}\right) \\
& \dot{N}_{2} / N_{b} \approx+\left(P_{M}\right) .
\end{aligned}
$$

This set of equations have a particular simple and interesting set of analytical solutions that we now explore before moving on. For this, we start by rewriting the
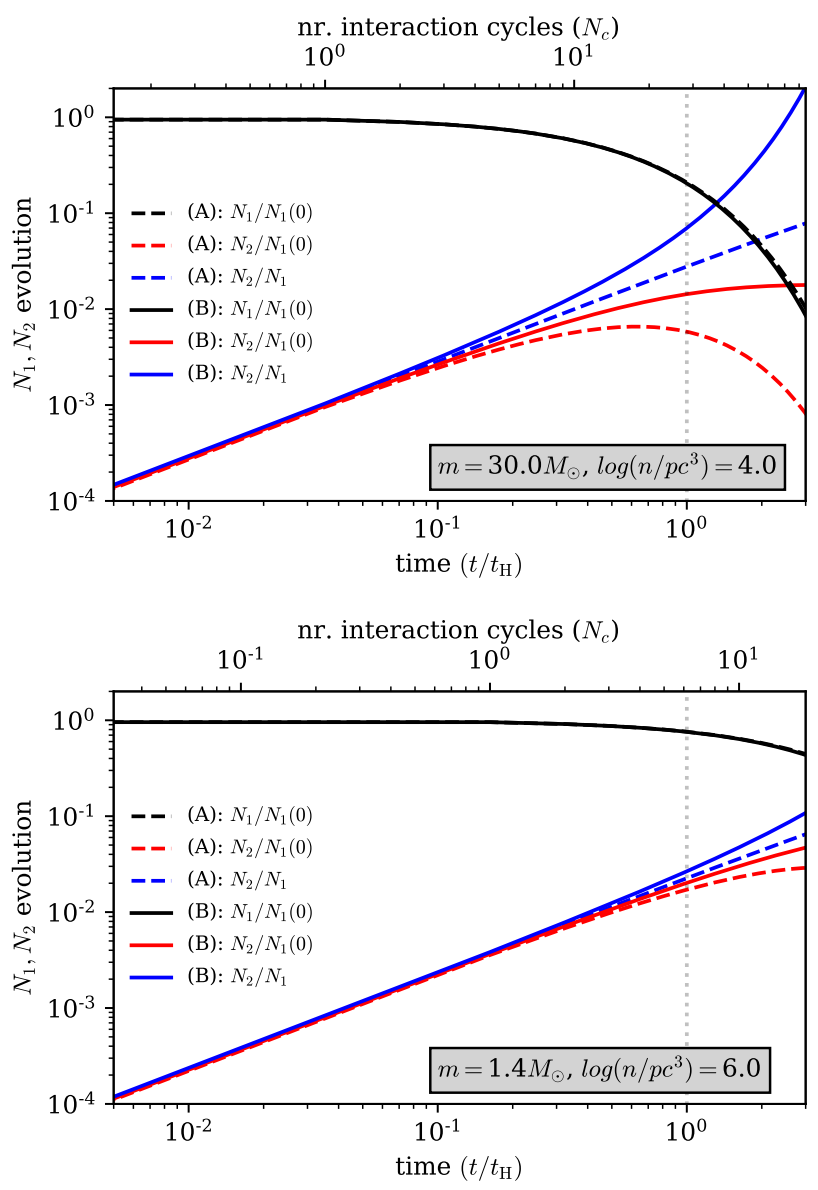

Figure 5. Re-scaled evolution of 1G-objects (black lines), $N_{1} / N_{1}(0), 2$ G-objects (red lines), $N_{2} / N_{1}(0)$, and their number ratio (blue lines), $N_{2} / N_{1}$, as a function of time (lower $x$-axis), $t / t_{H}$, and corresponding number of ICs (upper $x$ axis), $N_{c}=t / \tau_{m}$. The dashed lines and solid lines show the solution from our cases $c A$ and $c B$ described in Sec. 3.2, respectively. For both plots we have assumed that $f_{b}=0.01$, $N_{t}^{e j}=6, v_{d}=10 \mathrm{kms}^{-1}$, and $R_{m}^{11}=1$, where the upper plot shows results for $m=30 M_{\odot}$, and the lower plot for $m=1.4 M_{\odot}$. In the $m=30 M_{\odot}$ case the ratio $N_{2} / N_{1}$ approaches the $10 \%$-level at $t / t_{H} \sim 1$ for our chosen parameters, which indicates that populating the UMG through in-cluster 1G-1G GW mergers seems possible. This is in contrast to the $1.4 M_{\odot}$ case, where $n$ needs to take the relative high value of $n \sim 10^{6} p c^{-3}$ to even reach the $1 \%$-level. This is further discussed in Sec. 3.2 and Sec. 3.2.2.

above equations into a more general form to shorten the notations: $\dot{N}_{1}=-\alpha N_{b}, \dot{N}_{2}=\beta N_{b}$, where we have defined,

$$
\begin{aligned}
\alpha & =N_{t}^{e j}-P_{M} N_{s}^{e j} \\
\beta & =P_{M} .
\end{aligned}
$$

To proceed, we now consider a specific model where the binary fraction stays constant such that $N_{b}=f_{b} \times N_{1}$. In this case, the solution to the above set of equations 
is easily found from simple integrations, from which it follows,

$$
\begin{aligned}
& N_{1}=N_{1}(0) \times \exp \left(-\alpha f_{b} N_{c}\right) \\
& N_{2}=N_{1}(0) \times(\beta / \alpha)\left[1-\exp \left(-\alpha f_{b} N_{c}\right)\right],
\end{aligned}
$$

where $N_{1}(0)$ is the initial number of $1 \mathrm{G}$-objects, and $N_{c}=t / \tau_{m}$ is the number of ICs after time $t$. If we first consider the solution to $N_{1}$, we see that the population of $1 \mathrm{G}$-objects 'decays' over time as if the cluster represents a giant 'radioactive nuclei' with decay time $t_{c d}$, given by

$$
t_{c d} \approx \frac{\tau_{m}}{\alpha f_{b}},
$$

where the time for undergoing one IC, $\tau_{m}$, is given by Eq. (7). For example, for $a_{m}=a_{e j}$ the decay time is $t_{c d} \propto v_{d}^{3} /\left(n m^{2} f_{b}\right)$, where we have used Eq. (4). One consequence of this model is that the decay rate, and thereby the number of 1G-objects $N_{1}$ after a Hubble time, depends exponentially on the binary fraction $f_{b}$. The binary fraction is at the moment unknown observationally, but numerical simulations of GCs using Monte-Carlo techniques have shown that it very likely stays constant with only small scatter around $1-5 \%$ (see e.g. Fig. 2 in Samsing et al. 2019a). As a result, a significant fraction of present day GCs likely have many of their 1G-objects left in their core, where the remaining fraction have lost its BHs through binary-single 'evaporation'. This 'evaporation effect' will lead to a characteristic change in $\mathrm{BBH}$ merger rates as a function of redshift, similar to what is found for the set of GCs that 'evaporates' through tidal heating or direct tidal disruptions (e.g. Fragione \& Kocsis 2018). Considering now $N_{2}$ for our model, we see that at early times $N_{2} \approx N_{1}(0) f_{b} P_{M} N_{c}$, where we have used that $\exp (-a x) \approx 1-a x$. This is expected, as this simply equals the number of mergers per IC evaluated for the initial $N_{1}(0)$ population $\left(N_{1}(0) f_{b} P_{M}\right)$ times the number of ICs $\left(N_{c}\right)$. Note that this is similar to Eq. (19), where we studied how effective a population consisting of a single binary (' $1=N_{1}(0) f_{b}$ ') is at growing a 2G-population. As $N_{c}$ increases towards infinity, the $N_{2}$ population reaches a maximum 'freeze-out value', $\max \left(N_{2}\right)$, given by

$$
\max \left(N_{2}\right)=N_{1}(0)(\beta / \alpha), N_{c} \rightarrow \infty,
$$

which interestingly do not depend on the binary fraction, although how fast $N_{2}$ reaches $\max \left(N_{2}\right)$ does. As seen, within a factor of unity, $\max \left(N_{2}\right)$ its simply given by the total number of binary mergers one would get if one turned the initial $N_{1}(0)$ population into a total of $N_{1}(0) / 2$ binaries. Finally, if we now consider the number of $2 \mathrm{G}$-objects relative to $1 \mathrm{G}$-objects, one finds using
Eq. (37) that

$$
N_{2} / N_{1}=(\beta / \alpha)\left[\exp \left(\alpha f_{b} N_{c}\right)-1\right] .
$$

We see here that this ratio always increases, i.e., in this case there is no 'freeze-out' value. This of course originates from that $N_{1}$ keeps decreasing, whereas $N_{2}$ keeps increasing until it asymptotically reaches its value $\max \left(N_{2}\right)$. Considering the limit where $N_{2} / N_{1}=1$, we can solve for the corresponding characteristic $N_{c}$ scale, denoted here by $N_{c}^{2 E 1}$,

$$
N_{c}^{2 E 1}=\frac{\ln (1+\alpha / \beta)}{\alpha f_{b}},
$$

which equals the number of IC cycles, or time $t_{c}^{2 E 1} \approx$ $N_{c}^{2 E 1} \times \tau_{m}$, it takes for $N_{2}$ to be similar to $N_{1}$. Comparing $t_{c}^{2 E 1}$ with $t_{H}$ provides a rough estimate for when a system is effective in growing a sizable $2 \mathrm{G}$-population within a Hubble time. We will study the ratio $N_{2} / N_{1}$ from $c B$ in greater detail in Sec. 3.2.2 below.

The evolution of $N_{1}$ and $N_{2}$ for case $c A$ and $c B$ as a function of time is shown in Fig. 5 assuming the binary fraction stays constant at $f_{b}=0.01, N_{t}^{e j}=6, N_{s}^{e j}=4$, $v_{d}=10 \mathrm{kms}^{-1}$, and $R_{M}^{11}=1$. Note here that in the upper plot where $m=30 M_{\odot}$ the density is $n=10^{4} p c^{-3}$, whereas in the lower plot for $m=1.4 M_{\odot}$ the density is instead $n=10^{6} p c^{-3}$, as this is around the threshold for when $N_{c} \gg 1$ (see Fig. 2). Starting with $c A$, we see in the $m=30 M_{\odot}$ case how the 2G-population first grows steadily up to a given point just before $t=t_{H}$, after which it starts decreasing. This decrease is sourced by the binary and single ejection sink terms from Eq. (34). Considering now $N_{2} / N_{1}$, we see that at $t=t_{H}$ this ratio is (only) at the $1 \%$-level. We therefore expect $N_{2} / N_{1}$ to be of that order or greater for these cluster values, depending on the retention fraction $R_{M}^{11}$. The same characteristics are true for the $m=1.4 M_{\odot}$ case, but to reach a value for $N_{2} / N_{1}$ of a few percent, we see that $n$ in this case has to be of order $10^{6} p c^{-3}$, which is much higher than what is found in most astrophysical systems. Considering now $c B$, it is seen for $m=30 M_{\odot}$ that $N_{1}$ decays exponentially, whereas $N_{2}$ steadily levels off at its 'freeze-out value' given by Eq. (39). The characteristic time given by Eq. (41) for which $N_{2}=N_{1}$ is only $2 \sim 3$ times $t_{H}$, and as a result, the ratio $N_{2} / N_{1}$ approaches here the $10 \%$-level at $t_{H}$. This indicates that $\gtrsim 30 M_{\odot}$ COs are able to reach interesting limits when it comes to populating the UMG, whereas in the $\sim 1 M_{\odot}$ $\mathrm{CO}$ case, it seems very difficult to undergo enough incluster mergers to populate the LMG. We will study this in greater detail in the section below.

\subsubsection{Upper Limits on 2G-Objects}



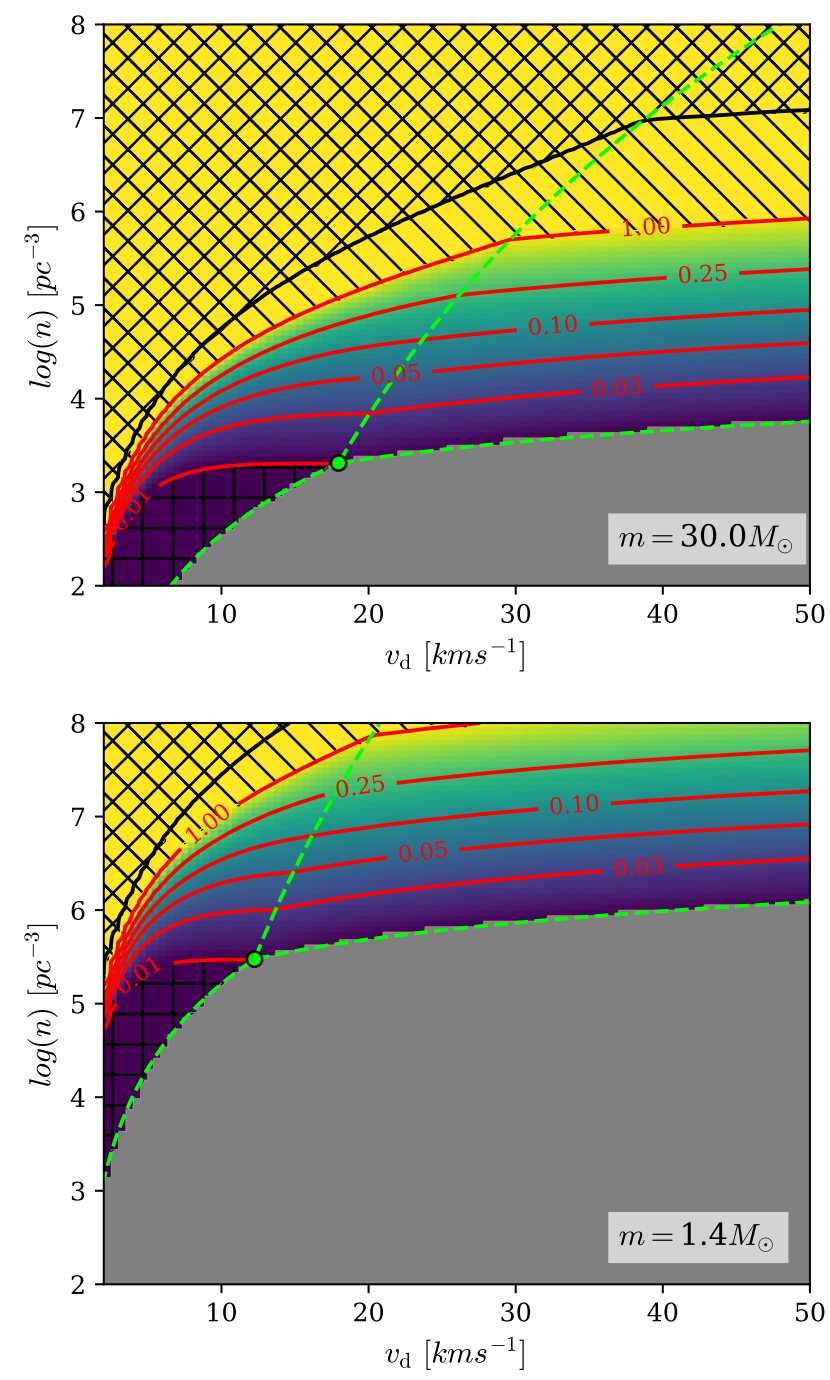

Figure 6. Results from our considered case $c B$ described in Sec. 3.2.1, where the number of $1 \mathrm{G}$ - and $2 \mathrm{G}$-object as a function of time is given by Eq. (37), and their ratio $N_{2} / N_{1}$ by Eq. (40). We here consider solutions to $t=t_{H}$ for a model described by $f_{b}=0.01, R_{M}^{11}=1, N_{t}^{e j}=6$, and $N_{s}^{e j}=4$, where the upper and lower plots correspond to $m=30 M_{\odot}$ and $m=1.4 M_{\odot}$, respectively. The area covered by the red contours is where $0.01<N_{2} / N_{1}<1.0$, i.e. it is the region that gives rise to both consistent $(<1.0)$ and interesting $(>$ 0.01 ) outcomes for growing a $2 \mathrm{G}$-population. In the yellow $1 \backslash$ '-hatched area, our formalism evaluated at $t=t_{H}$ breaks down as $N_{2}$ is here $>N_{1}$, where in the grey area our $N_{c}$ averaging approach breaks down as $N_{c}$ is here $<1$. In the yellow ' $X$ '-hatched area $N_{1} / N_{1}(0)<10^{-4}$; therefore, if a system is located within this area it will 'evaporate' within a Hubble time if its initial number of BHs is $\lesssim 10^{4}$. The '+'-hatched area is where $N_{2} / N_{1}<0.01$ and $N_{c} \gg 1$, and highlights therefore systems that clearly undergo several ICs, but still end up with a relative small 2G-population. The green separation lines are describe in Sec. 2.2.1. Results related to this figure are described in Sec. 3.2.2.
Fig. 6 shows results related to the ratio $N_{2} / N_{1}$ given by Eq. (40) evaluated at $t=t_{H}$, as further described in the figure caption. As described in Sec. 3.2.1, this case represents in our model an upper limit on $N_{2} / N_{1}$. Considering first the upper plot showing the $m=30 M_{\odot}$ case, we see that for a GC with $v_{d} \sim 10 \mathrm{kms}^{-1}$ a population of $1 \mathrm{G}$-objects can over a Hubble time turn into a population with $N_{2} / N_{1}>0.1$ if $n \gtrsim 10^{4} p c^{-3}$. Although this is an upper limit, it greatly illustrates that the length, mass, and times scales associated with a typical cluster hosting $\mathrm{BHs}$ of mass $\sim 30 M_{\odot}$ in the core is able to populate the upper mass gap through successive mergers of its 1G-population. Considering now the lower plot showing results for the $1.4 M_{\odot}$ case, we see that for $v_{d} \sim 10 \mathrm{kms}^{-1}$ the density has to be $\gtrsim 10^{5} \mathrm{pc}^{-3}$ to even grow a $2 \mathrm{G}$-population with $N_{2} / N_{1}>0.01$, and $\gtrsim 10^{6} p c^{-3}$ for $N_{2} / N_{1}>0.1$. From this we conclude that populating the lower mass gap through successive mergers of NSs in any reasonable astrophysical cluster seems almost impossible, not even when we assume that the entire population is consisting of only NSs. This last assumption is in fact also highly optimistic, as NSs will not segregate and form their own sub-cluster in the same way as $\mathrm{BHs}$ because their characteristic $1.4 M_{\odot}$ mass is very close to that of the ordinary stars in the cluster. As a result, NSs will exchange and interact frequently with the stellar population, which introduces 'impurities' in the IC illustrated in Fig. 1. The probability that two NSs merge inside the cluster is therefore significantly smaller than what we have assumed in our considered $c B$ scenario. In comparison, the $\mathrm{BHs}$ have such a large mass compared to the remaining stellar population, that they easily form their own sub-system (e.g. Askar et al. 2018). In Fig. 7 we show how these results depend more broadly on the mass $m$, where we show $N_{2} / N_{1}$ from case $c B$, as a function of $m$ for $n=10^{4} p c^{-3}$ (top plot) and $n=10^{5} p c^{-3}$ (bottom plot), and two different binary fractions, as further described in the figure caption.

Finally, we note that the real 'bottle neck' in populating the lower mass gap is not directly related to the probability $P_{M}$ per IC for a NS population to undergo NS-NS mergers inside their cluster. Instead, it is the time it takes for a NS-NS binary to undergo one IC, $\tau_{m}$, that simply is too long for a standard cluster. This is clear from Fig. 6 , as the grey area, where $N_{c} \lesssim 1$, sets the lower limit at $n=10^{5} \mathrm{pc}^{-3}$ for $10 \mathrm{kms}^{-1}$. In the limit where $a_{m}=a_{e j}$ the number of ICs evaluated at 
$t_{H}, N_{c}\left(t_{H}\right)=t_{H} / \tau_{m}\left(a_{e j}\right)$, is given by,

$$
\begin{aligned}
N_{c}\left(t_{H}\right) & \approx t_{H}\left[\frac{\pi G^{2} \Delta^{2}}{\delta f_{e d}^{2}}\right] \times \frac{n m^{2}}{v_{d}^{3}} \\
& \approx 0.8\left(\frac{n}{10^{5} p c^{-3}}\right)\left(\frac{m}{1.4 M_{\odot}}\right)^{2}\left(\frac{v_{d}}{10 k m s^{-1}}\right)^{-3}
\end{aligned}
$$

and is indeed just around unity for NS-NS binaries for our chosen normalizations. It is furthermore seen that $N_{c}\left(t_{H}\right)$ rapidly decreases with mass $m$ as $\propto m^{2}$. However, as seen on Fig. 3, if the system is in the area for which $N_{c}\left(t_{H}\right)>1$, the dependence on $m$ on how many in-cluster mergers a given binary can produce within a Hubble time, $N_{M}^{\prime}\left(t_{H}\right)$, is less sensitive to $m$, as $N_{c}\left(t_{H}\right) \times P_{m} \propto m^{8 / 7}$. All in all, the limit for which $N_{c}\left(t_{H}\right)=1$ plays therefore a crucial role for determining what systems that are able to produce a significant 2G-population. We conclude our study below.

\section{CONCLUSIONS}

We have in this paper studied the formation of $2 \mathrm{G}$ objects formed through $1 \mathrm{G}-1 \mathrm{G}$ in-cluster mergers in dense clusters. We have in particular explored the possibility for populating the LMG $\left(3-5 M_{\odot}\right)$ and the UMG ( $245 M_{\odot}$ ) through the merger of BNSs and BBHs, respectively. Understanding what cluster systems that are able to populate these two mass-gaps has wide implications for both GW astrophysics and stellar physics. For example, if nature is proven not to be able to create mass-gap BHs through normal stellar evolution, then current and future measures of the BH mass spectrum, through e.g. GW observations, will give us insight into the formation mechanisms of BBH mergers in clusters. On the other hand, if observations hint that stellar clusters do not contribute significantly to the observed GW merger rate, e.g. through independent measures of the fraction of eccentric BBH mergers (e.g. Samsing 2018), then an observed population of mass-gap objects will hint that our single stellar models need to be revised. For these reasons, several new studies have discussed the possibility for dynamically populating these mass gaps (e.g. O'Leary et al. 2016; Fishbach et al. 2017; Gerosa \& Berti 2017; Yang et al. 2019; Antonini et al. 2019; Gerosa \& Berti 2019; Samsing \& Ilan 2019; Rodriguez et al. 2019; Gerosa et al. 2020; Safarzadeh et al. 2020; Gayathri et al. 2020; Kimball et al. 2020; Doctor et al. 2020; Baibhav et al. 2020).

Through a fully analytical approach we have here studied how efficient a cluster, described by a constant $v_{d}, n$, can turn its initial population of $N_{1} 1 \mathrm{G}$-objects into a sizable population of $N_{2}$ 2G-objects through incluster GW mergers. We have in particular explored the
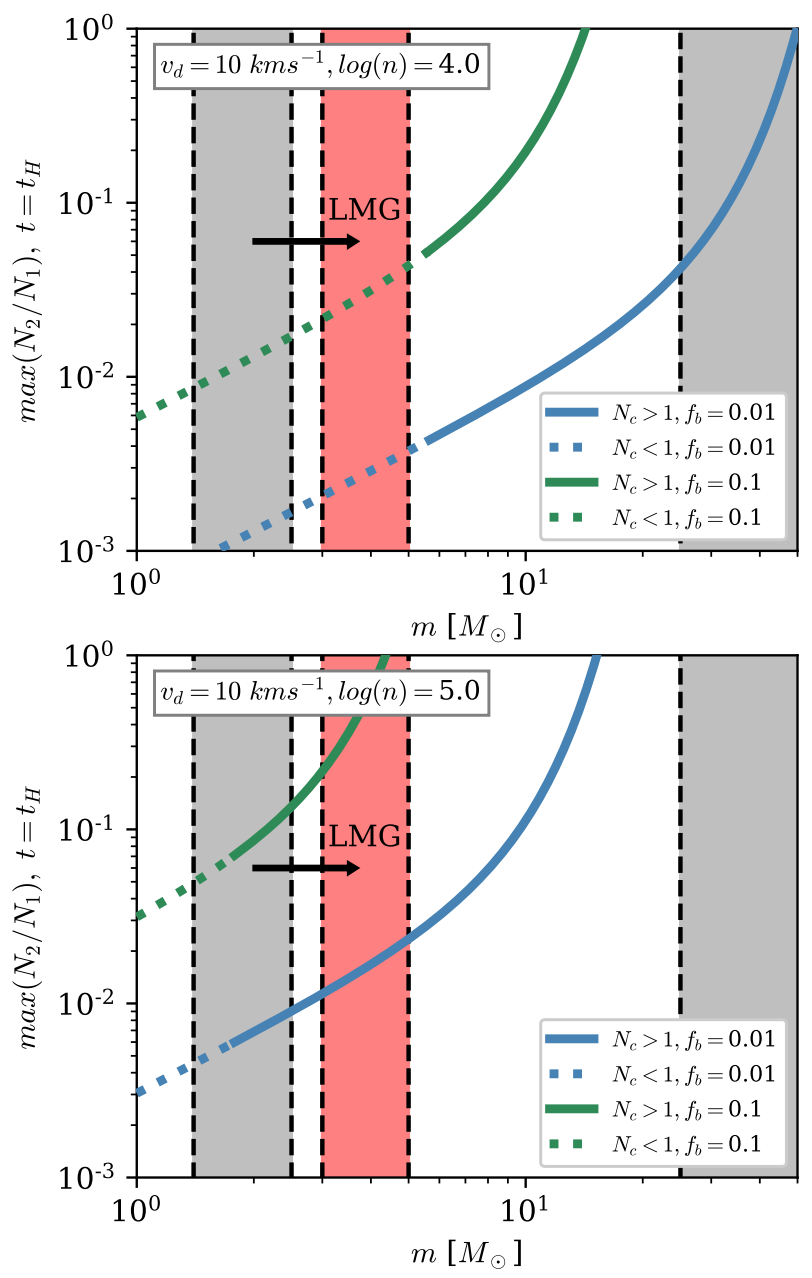

Figure 7. Number of $2 \mathrm{G}$-objects $\left(N_{2}\right)$ relative to $1 \mathrm{G}$-objects $\left(N_{1}\right)$ derived for our case $c B$ using Eq. (40) at $t=t_{H}$, as a function of $m$ for fixed $v_{d}$, but varying $n$ and $f_{b}$, as further indicated in the legends. The two plots differ by the value of $n$, where $n=10^{4} p c^{-3}$ and $n=10^{5} p c^{-3}$ in the upper and lower plots, respectively. The grey bands show the mass range for which a merger that produces a remnant with mass $\sim 2 m$ will land in the corresponding mass gap, where the red band shows the LMG (the UMG is not shown). For example, a merger between two COs (NSs) in the lower grey band will form a merger product that lands in the red LMG band, as further illustrated by the black arrow. The dotted lines highlight the part of the curves for which $N_{c}<1$ ( $2 \mathrm{G}$ formation is highly ineffective), where the solid lines correspond to $N_{c}>1$ ( $2 \mathrm{G}$ formation is possible).

upper limit on the ratio $N_{2} / N_{1}$ evaluated after a Hubble time, as a function of $v_{d}, n$ and $m$ (Sec. 3.2.2). Our limit is based entirely on dynamics, and complements therefore greatly the recent study by Gerosa \& Berti (2019), where the limit was derived from considering the magnitude of GW kicks. From our analysis we have reached the following conclusions: 
Populating the LMG through in-cluster mergers of BNSs is a very slow process for any astrophysical cluster. For example, as shown in Fig. 6, even in the highly idealized case of a GC core populated entirely by NSs, the number density $n$ has to be $>10^{6} p c^{-3}$ to reach $N_{2} / N_{1} \sim 0.1$. As discussed in Sec. 3.2.2, not only is this density much higher than what is found for real clusters, but NSs are also likely to mix with other stars due to their similar mass, which reduces their in-cluster merger probability further. In fact, our results show that what really limits a NS rich core to undergo enough in-cluster mergers to populate the LMG is actually the timescale for interactions, and not how the NSs exactly merge inside their cluster. This is seen in Fig. 6, where for a NS dominated core (bottom plot) a density of $n \gtrsim 10^{5} p c^{-3}$ for $v_{d} \sim 10 \mathrm{kms}^{-1}$ is required to move above the grey area, i.e. for a BNS to undergo at least 1 IC. In our described 'standard picture' of dynamically assembled in-cluster mergers (Sec. 2.1), an efficient production of LMG objects is therefore highly unlikely. If clusters for some reason are still observed to effectively produce LMG objects through dynamics, then more 'exotic' dynamical pathways have to be evoked. Alternatively, it could be that some clusters start out with a high BNS fraction (see Fig. 7) that would lead to a relative high number of $2 \mathrm{G}$ objects after a Hubble time. However, in that case, there would still be problems related to how fast this $2 \mathrm{G}$ population can be dynamically paired up with other COs to undergo, say, observable GW mergers. Therefore, observing GW sources with at least one LMG object formed in a cluster near the grey area in Fig. 6 (bottom) seems therefore highly unlikely.

Populating the UMG is in comparison much easier, e.g., in Fig. 6 (top) it is clearly seen that reaching values of $N_{2} / N_{1} \sim 0.1$ only requires clusters with a central density of $\sim 10^{4} p c^{-3}$. This is a much more reasonable magnitude, which leads us to conclude that populating the UMG in clusters is relatively easy, at least dynamically, without introducing any non-standard pathways. Our model even implies that for $n \gtrsim 10^{4} p c^{-3}$ there is high probability for the initial $1 \mathrm{G}$ population to turn almost entirely into a $2 \mathrm{G}$ population. Our models are not able to accurately describe this scenario, but it does at least hint that in moderate dense clusters in-cluster mergers can be highly effective in changing the initial mass function. This has great implications for 3. generation GW observations where will see every BBH merger within our observable patch as a function of redshift.

Finally, we note that a few studies that were completed while our present study was underway point towards similar conclusions to what we have her. For example, in Ye et al. (2020) it was shown using a fully numerical approach that the rate of BNS mergers originating from GCs is low, where both Rodriguez et al. (2019) and Baibhav et al. (2020) illustrated that populating the UMG definitely seems possible. However, other studies still keep the question open to what degree the LMG can be populated in clusters (e.g. Gupta et al. 2020). The topic is therefore highly rich and interesting, and our study greatly compliments this recent literature with the first set of closed form solutions that encapsulate all the correct scalings and relations of the problem. We note here that standard brute-force $N$ body techniques are still too slow at evolving high density clusters, which is why we and others explore how to solve this problem using approximate schemes (see also work by Antonini et al. (2019); Antonini \& Gieles (2020)). We are currently working on a self consistent hybrid scheme that will enable us to correctly evolve a full mass distribution. Our present paper plays a crucial role in providing the first steps in this highly relevant and timely topic.

\section{ACKNOWLEDGMENTS}

The authors thank the Yukawa Institute for Theoretical Physics at Kyoto University, and the organizers of the workshop YKIS2019 'Black Holes and Neutron Stars with Gravitational Waves', where many useful conversations took place. It is also a pleasure to thank Kyle Kremer for enlightening discussions. JS acknowledges support from the Lyman Spitzer Fellowship and the European Unions Horizon 2020 research and innovation programme under the Marie Sklodowska-Curie grant agreement No. 844629. KH acknowledges support from the Lyman Spitzer Fellowship.

\section{REFERENCES}

Aarseth, S. J., \& Heggie, D. C. 1976, A\&A, 53, 259

Abbott, B. P., Abbott, R., Abbott, T. D., et al. 2016a,

Physical Review Letters, 116, 061102,

doi: 10.1103/PhysRevLett.116.061102
—. 2016b, Physical Review Letters, 116, 241103,

doi: 10.1103/PhysRevLett.116.241103

—. 2016c, Physical Review X, 6, 041015, doi: 10.1103/PhysRevX.6.041015 
—. 2017a, Physical Review Letters, 118, 221101, doi: 10.1103/PhysRevLett.118.221101

—. 2017b, Physical Review Letters, 119, 141101, doi: 10.1103/PhysRevLett.119.141101

—. 2017c, Physical Review Letters, 119, 161101, doi: 10.1103/PhysRevLett.119.161101

Andrews, J. J., \& Mandel, I. 2019, ApJL, 880, L8, doi: $10.3847 / 2041-8213 /$ ab2ed1

Antonini, F., Chatterjee, S., Rodriguez, C. L., et al. 2016, ApJ, 816, 65, doi: 10.3847/0004-637X/816/2/65

Antonini, F., \& Gieles, M. 2020, MNRAS, 492, 2936, doi: $10.1093 / \mathrm{mnras} / \mathrm{stz} 3584$

Antonini, F., Gieles, M., \& Gualandris, A. 2019, MNRAS, 486, 5008, doi: 10.1093/mnras/stz1149

Antonini, F., \& Rasio, F. A. 2016, ApJ, 831, 187, doi: 10.3847/0004-637X/831/2/187

Antonini, F., Rodriguez, C. L., Petrovich, C., \& Fischer, C. L. 2018, MNRAS, 480, L58, doi: $10.1093 / \mathrm{mnrasl} / \mathrm{sly} 126$

Antonini, F., Toonen, S., \& Hamers, A. S. 2017, ApJ, 841, 77, doi: 10.3847/1538-4357/aa6f5e

Askar, A., Arca Sedda, M., \& Giersz, M. 2018, MNRAS, 478, 1844, doi: 10.1093/mnras/sty1186

Askar, A., Szkudlarek, M., Gondek-Rosińska, D., Giersz, M., \& Bulik, T. 2017, MNRAS, 464, L36, doi: $10.1093 / \mathrm{mnrasl} / \mathrm{slw} 177$

Bae, Y.-B., Kim, C., \& Lee, H. M. 2014, MNRAS, 440, 2714, doi: 10.1093/mnras/stu381

Baibhav, V., Gerosa, D., Berti, E., et al. 2020, arXiv e-prints, arXiv:2004.00650. https://arxiv.org/abs/2004.00650

Bailyn, C. D., Jain, R. K., Coppi, P., \& Orosz, J. A. 1998, ApJ, 499, 367, doi: 10.1086/305614

Banerjee, S., Baumgardt, H., \& Kroupa, P. 2010, MNRAS, 402, 371, doi: 10.1111/j.1365-2966.2009.15880.x

Bartos, I., Kocsis, B., Haiman, Z., \& Márka, S. 2017, ApJ, 835, 165, doi: 10.3847/1538-4357/835/2/165

Belczynski, K., Holz, D. E., Bulik, T., \& O'Shaughnessy, R. 2016a, Nature, 534, 512, doi: 10.1038/nature18322

Belczynski, K., Repetto, S., Holz, D. E., et al. 2016b, ApJ, 819, 108, doi: 10.3847/0004-637X/819/2/108

Berti, E., Cardoso, V., Gonzalez, J. A., et al. 2007, PhRvD, 76, 064034, doi: 10.1103/PhysRevD.76.064034

Bird, S., Cholis, I., Muñoz, J. B., et al. 2016, Physical Review Letters, 116, 201301, doi: 10.1103/PhysRevLett.116.201301

Carr, B., Kühnel, F., \& Sandstad, M. 2016, PhRvD, 94, 083504, doi: 10.1103/PhysRevD.94.083504

Chen, X., \& Amaro-Seoane, P. 2017, ApJL, 842, L2, doi: $10.3847 / 2041-8213 /$ aa74ce
Cholis, I., Kovetz, E. D., Ali-Haïmoud, Y., et al. 2016, PhRvD, 94, 084013, doi: 10.1103/PhysRevD.94.084013

Doctor, Z., Wysocki, D., O'Shaughnessy, R., Holz, D. E., \& Farr, B. 2020, ApJ, 893, 35, doi: $10.3847 / 1538-4357 / \mathrm{ab} 7 \mathrm{fac}$

Dominik, M., Belczynski, K., Fryer, C., et al. 2012, ApJ, 759, 52, doi: 10.1088/0004-637X/759/1/52

—. 2013, ApJ, 779, 72, doi: 10.1088/0004-637X/779/1/72

Dominik, M., Berti, E., O'Shaughnessy, R., et al. 2015, ApJ, 806, 263, doi: 10.1088/0004-637X/806/2/263

D'Orazio, D. J., \& Loeb, A. 2017, ArXiv e-prints. https://arxiv.org/abs/1706.04211

Farmer, R., Renzo, M., de Mink, S. E., Marchant, P., \& Justham, S. 2019, ApJ, 887, 53, doi: 10.3847/1538-4357/ab518b

Farr, W. M., Fishbach, M., Ye, J., \& Holz, D. E. 2019, ApJL, 883, L42, doi: 10.3847/2041-8213/ab4284

Farr, W. M., Sravan, N., Cantrell, A., et al. 2011, ApJ, 741, 103, doi: 10.1088/0004-637X/741/2/103

Fishbach, M., Holz, D. E., \& Farr, B. 2017, ApJL, 840, L24, doi: 10.3847/2041-8213/aa7045

Fragione, G., \& Bromberg, O. 2019, arXiv e-prints, arXiv:1903.09659. https://arxiv.org/abs/1903.09659

Fragione, G., \& Kocsis, B. 2018, PhRvL, 121, 161103, doi: 10.1103/PhysRevLett.121.161103

—. 2019, MNRAS, 486, 4781, doi: 10.1093/mnras/stz1175

—. 2020, MNRAS, 493, 3920, doi: 10.1093/mnras/staa443

Fragione, G., \& Loeb, A. 2019, MNRAS, 486, 4443, doi: 10.1093/mnras/stz1131

Gayathri, V., Bartos, I., Haiman, Z., et al. 2020, ApJL, 890, L20, doi: 10.3847/2041-8213/ab745d

Gerosa, D., \& Berti, E. 2017, PhRvD, 95, 124046, doi: 10.1103/PhysRevD.95.124046

—. 2019, PhRvD, 100, 041301, doi: 10.1103/PhysRevD.100.041301

Gerosa, D., Vitale, S., \& Berti, E. 2020, arXiv e-prints, arXiv:2005.04243. https://arxiv.org/abs/2005.04243

Giersz, M., Leigh, N., Hypki, A., Lützgendorf, N., \& Askar, A. 2015, MNRAS, 454, 3150, doi: 10.1093/mnras/stv2162

Gültekin, K., Miller, M. C., \& Hamilton, D. P. 2004, ApJ, 616,221

—. 2006, ApJ, 640, 156

Gupta, A., Gerosa, D., Arun, K. G., et al. 2020, PhRvD, 101, 103036, doi: 10.1103/PhysRevD.101.103036

Hamers, A. S., Bar-Or, B., Petrovich, C., \& Antonini, F. 2018, ApJ, 865, 2, doi: 10.3847/1538-4357/aadae2

Hamers, A. S., \& Samsing, J. 2019a, MNRAS, 487, 5630, doi: 10.1093/mnras/stz1646

—. 2019b, MNRAS, 488, 5192, doi: 10.1093/mnras/stz2029

—. 2020, MNRAS, 494, 850, doi: 10.1093/mnras/staa691 
Hamers, A. S., \& Thompson, T. A. 2019, ApJ, 883, 23, doi: 10.3847/1538-4357/ab3b06

Heggie, D. C. 1975, MNRAS, 173, 729

Hénault-Brunet, V., Gieles, M., Strader, J., et al. 2020, MNRAS, 491, 113, doi: 10.1093/mnras/stz2995

Hoang, B.-M., Naoz, S., Kocsis, B., Rasio, F. A., \& Dosopoulou, F. 2017, ArXiv e-prints. https://arxiv.org/abs/1706.09896

Hong, J., \& Lee, H. M. 2015, MNRAS, 448, 754, doi: $10.1093 / \mathrm{mnras} / \mathrm{stv} 035$

Hotokezaka, K., \& Piran, T. 2017, ApJ, 842, 111, doi: 10.3847/1538-4357/aa6f61

Hut, P., \& Bahcall, J. N. 1983, ApJ, 268, 319

Janiuk, A., Bejger, M., Charzyński, S., \& Sukova, P. 2017, ArXiv e-prints, 51, 7, doi: 10.1016/j.newast.2016.08.002

Kalogera, V. 2000, ApJ, 541, 319, doi: 10.1086/309400

Kimball, C., Talbot, C., Berry, C. P. L., et al. 2020, arXiv e-prints, arXiv:2005.00023.

https://arxiv.org/abs/2005.00023

Kinugawa, T., Inayoshi, K., Hotokezaka, K., Nakauchi, D., \& Nakamura, T. 2014, MNRAS, 442, 2963, doi: 10.1093/mnras/stu1022

Kizıltan, B., Baumgardt, H., \& Loeb, A. 2017, Nature, 542, 203, doi: 10.1038/nature21361

Kremer, K., Lu, W., Rodriguez, C. L., Lachat, M., \& Rasio, F. A. 2019a, ApJ, 881, 75, doi: 10.3847/1538-4357/ab2e0c

Kremer, K., Ye, C. S., Chatterjee, S., Rodriguez, C. L., \& Rasio, F. A. 2020, in IAU Symposium, Vol. 351, IAU Symposium, ed. A. Bragaglia, M. Davies, A. Sills, \& E. Vesperini, 357-366, doi: 10.1017/S1743921319007269

Kremer, K., Rodriguez, C. L., Amaro-Seoane, P., et al. 2019b, PhRvD, 99, 063003, doi: 10.1103/PhysRevD.99.063003

Leung, S.-C., Nomoto, K., \& Blinnikov, S. 2019, ApJ, 887, 72, doi: 10.3847/1538-4357/ab4fe5

Liu, B., \& Lai, D. 2017, ApJL, 846, L11, doi: $10.3847 / 2041-8213 /$ aa8727

—. 2018, ApJ, 863, 68, doi: 10.3847/1538-4357/aad09f

—. 2019, MNRAS, 483, 4060, doi: 10.1093/mnras/sty3432

Liu, B., Lai, D., \& Wang, Y.-H. 2019, ApJ, 881, 41, doi: $10.3847 / 1538-4357 / \mathrm{ab} 2 \mathrm{dfb}$

Loeb, A. 2016, ApJL, 819, L21, doi: $10.3847 / 2041-8205 / 819 / 2 /$ L21

Lopez, Martin, J., Batta, A., Ramirez-Ruiz, E., Martinez, I., \& Samsing, J. 2019, ApJ, 877, 56, doi: $10.3847 / 1538-4357 /$ ab1842

McKernan, B., Ford, K. E. S., Bellovary, J., et al. 2017, ArXiv e-prints. https://arxiv.org/abs/1702.07818

Miller, M. C., \& Davies, M. B. 2012, ApJ, 755, 81, doi: 10.1088/0004-637X/755/1/81
Murguia-Berthier, A., MacLeod, M., Ramirez-Ruiz, E., Antoni, A., \& Macias, P. 2017, ApJ, 845, 173, doi: $10.3847 / 1538-4357 /$ aa 8140

Naoz, S. 2016, ARA\&A, 54, 441, doi: 10.1146/annurev-astro-081915-023315

Naoz, S., Kocsis, B., Loeb, A., \& Yunes, N. 2013, ApJ, 773, 187, doi: 10.1088/0004-637X/773/2/187

O'Leary, R. M., Kocsis, B., \& Loeb, A. 2009, MNRAS, 395, 2127, doi: 10.1111/j.1365-2966.2009.14653.x

O'Leary, R. M., Meiron, Y., \& Kocsis, B. 2016, ApJL, 824, L12, doi: 10.3847/2041-8205/824/1/L12

Özel, F., Psaltis, D., Narayan, R., \& McClintock, J. E. 2010, ApJ, 725, 1918, doi: 10.1088/0004-637X/725/2/1918

Park, D., Kim, C., Lee, H. M., Bae, Y.-B., \& Belczynski, K. 2017, MNRAS, 469, 4665, doi: 10.1093/mnras/stx1015

Piran, Z., \& Piran, T. 2020, ApJ, 892, 64, doi: $10.3847 / 1538-4357 / \mathrm{ab} 792 \mathrm{a}$

Portegies Zwart, S. F., \& McMillan, S. L. W. 2000, ApJ, 528, L17

Randall, L., \& Xianyu, Z.-Z. 2018a, ApJ, 864, 134, doi: 10.3847/1538-4357/aad7fe

—. 2018b, ApJ, 853, 93, doi: 10.3847/1538-4357/aaa1a2

Rodriguez, C. L., Amaro-Seoane, P., Chatterjee, S., et al. 2018, PhRvD, 98, 123005, doi: 10.1103/PhysRevD.98.123005

Rodriguez, C. L., \& Antonini, F. 2018, ApJ, 863, 7, doi: $10.3847 / 1538-4357 /$ aacea4

Rodriguez, C. L., Chatterjee, S., \& Rasio, F. A. 2016a, PhRvD, 93, 084029, doi: 10.1103/PhysRevD.93.084029

Rodriguez, C. L., Haster, C.-J., Chatterjee, S., Kalogera, V., \& Rasio, F. A. 2016b, ApJL, 824, L8, doi: 10.3847/2041-8205/824/1/L8

Rodriguez, C. L., Morscher, M., Pattabiraman, B., et al. 2015, Physical Review Letters, 115, 051101, doi: 10.1103/PhysRevLett.115.051101

Rodriguez, C. L., Zevin, M., Amaro-Seoane, P., et al. 2019, PhRvD, 100, 043027, doi: 10.1103/PhysRevD.100.043027

Rodriguez, C. L., Zevin, M., Pankow, C., Kalogera, V., \& Rasio, F. A. 2016c, ApJL, 832, L2, doi: 10.3847/2041-8205/832/1/L2

Safarzadeh, M., Hamers, A. S., Loeb, A., \& Berger, E. 2020, ApJL, 888, L3, doi: 10.3847/2041-8213/ab5dc8

Samsing, J. 2018, PhRvD, 97, 103014, doi: 10.1103/PhysRevD.97.103014

Samsing, J., Askar, A., \& Giersz, M. 2018a, ApJ, 855, 124, doi: $10.3847 / 1538-4357 /$ aaab52

Samsing, J., \& D'Orazio, D. J. 2018, MNRAS, doi: 10.1093/mnras/sty2334 
Samsing, J., D’Orazio, D. J., Kremer, K., Rodriguez, C. L., \& Askar, A. 2019a, arXiv e-prints, arXiv:1907.11231. https://arxiv.org/abs/1907.11231

Samsing, J., Hamers, A. S., \& Tyles, J. G. 2019b, PhRvD, 100, 043010, doi: 10.1103/PhysRevD.100.043010

Samsing, J., \& Ilan, T. 2018, MNRAS, 476, 1548, doi: $10.1093 / \mathrm{mnras} /$ sty 197

—. 2019, MNRAS, 482, 30, doi: 10.1093/mnras/sty2249

Samsing, J., MacLeod, M., \& Ramirez-Ruiz, E. 2014, ApJ, 784, 71, doi: 10.1088/0004-637X/784/1/71

—. 2018b, ApJ, 853, 140, doi: 10.3847/1538-4357/aaa715

Samsing, J., \& Ramirez-Ruiz, E. 2017, ApJL, 840, L14, doi: $10.3847 / 2041-8213 /$ aa6f0b

Samsing, J., Venumadhav, T., Dai, L., et al. 2019c, PhRvD, 100, 043009, doi: 10.1103/PhysRevD.100.043009

Sasaki, M., Suyama, T., Tanaka, T., \& Yokoyama, S. 2016, Physical Review Letters, 117, 061101, doi: 10.1103/PhysRevLett.117.061101

Schrøder, S. L., Batta, A., \& Ramirez-Ruiz, E. 2018, ApJL, 862, L3, doi: 10.3847/2041-8213/aacf8d

Sigurdsson, S., \& Phinney, E. S. 1993, ApJ, 415, 631

Silsbee, K., \& Tremaine, S. 2017, ApJ, 836, 39, doi: $10.3847 / 1538-4357 /$ aa5729

Stephan, A. P., Naoz, S., Ghez, A. M., et al. 2016, MNRAS, 460, 3494, doi: 10.1093/mnras/stw1220

Stone, N. C., \& Leigh, N. W. C. 2019, Nature, 576, 406, doi: 10.1038/s41586-019-1833-8

Stone, N. C., Metzger, B. D., \& Haiman, Z. 2017, MNRAS, 464, 946, doi: 10.1093/mnras/stw2260
Tagawa, H., Haiman, Z., \& Kocsis, B. 2019, arXiv e-prints, arXiv:1912.08218. https://arxiv.org/abs/1912.08218

Tanikawa, A. 2013, MNRAS, 435, 1358, doi: $10.1093 / \mathrm{mnras} / \mathrm{stt} 1380$

Toonen, S., Hamers, A., \& Portegies Zwart, S. 2016, Computational Astrophysics and Cosmology, 3, 6, doi: 10.1186/s40668-016-0019-0

VanLandingham, J. H., Miller, M. C., Hamilton, D. P., \& Richardson, D. C. 2016, ApJ, 828, 77, doi: $10.3847 / 0004-637 \mathrm{X} / 828 / 2 / 77$

Venumadhav, T., Zackay, B., Roulet, J., Dai, L., \& Zaldarriaga, M. 2019, arXiv e-prints, arXiv:1904.07214. https://arxiv.org/abs/1904.07214

Woosley, S. E. 2016, ApJL, 824, L10, doi: $10.3847 / 2041-8205 / 824 / 1 / \mathrm{L} 10$

—. 2017, ApJ, 836, 244, doi: 10.3847/1538-4357/836/2/244

Yang, Y., Bartos, I., Gayathri, V., et al. 2019, PhRvL, 123, 181101, doi: 10.1103/PhysRevLett.123.181101

Ye, C. S., Fong, W.-f., Kremer, K., et al. 2020, ApJL, 888, L10, doi: 10.3847/2041-8213/ab5dc5

Zackay, B., Venumadhav, T., Dai, L., Roulet, J., \& Zaldarriaga, M. 2019, arXiv e-prints, arXiv:1902.10331. https://arxiv.org/abs/1902.10331

Zaldarriaga, M., Kushnir, D., \& Kollmeier, J. A. 2018, MNRAS, 473, 4174, doi: 10.1093/mnras/stx2577

Zevin, M., Pankow, C., Rodriguez, C. L., et al. 2017, ApJ, 846, 82, doi: 10.3847/1538-4357/aa8408

Zevin, M., Samsing, J., Rodriguez, C., Haster, C.-J., \& Ramirez-Ruiz, E. 2019, ApJ, 871, 91, doi: $10.3847 / 1538-4357 /$ aaf6ec 